\title{
Haar Wavelet Expansions of Signals and their Applications in Image Processing
}

\author{
Shyam Lal \\ Department of Mathematics \\ Institute of Science \\ Banaras Hindu University \\ Varanasi-221005, India
}

\author{
K.K.Shukla \\ Department of Computer Science and Engineering \\ Indian Institute of Technology \\ Banaras Hindu University \\ Varanasi-221005, India
}

\author{
Sarika Keshri \\ DST-CIMS \\ Institute of Science \\ Banaras Hindu University \\ Varanasi-221005, India
}

\begin{abstract}
In this paper, double wavelet series of a signal $f$ of two variables $t_{1}$ and $t_{2}$ using Haar Scaling function $\Phi\left(t_{1}, t_{2}\right)=\phi\left(t_{1}\right) \phi\left(t_{2}\right)$ and Haar Wavelet function $\Psi\left(t_{1}, t_{2}\right)=\psi\left(t_{1}\right) \psi\left(t_{2}\right)$ has been introduced and it has been verified by a number of examples. Several properties of this signal and it's image have been studied.The significient result of this paper are the decomposition and reconstruction of signals of a single variable $t_{1}$ and signals of two variables $t_{1}$ and $t_{2}$ using Haar Scaling signal as well as Haar Wavelets.
\end{abstract}

\section{General Terms}

Multiresolution Analysis (MRA), Scaling function, Wavelets, Double Wavelet Series of two variables, Application of Wavelets in Image Processing.

\section{Keywords}

Haar Wavelet, Signal Processing, Image Processing, Double Wavelet Series, Signals of Lip $\alpha$ Class.

\section{INTRODUCTION}

The Haar Wavelet expansions of signal and their applications in Image Processing is an interesting problem in Computer Science. Haar Wavelet is generally known as the first basic wavelet. It was firstly introduced by Alfred Haar[1] in 1910. Haar Wavelets in single variable are applicable very frequently in Signal Processing. There is a need of larger storage space for the digital images because they have repeated data. Also, there is a requirement of much storage space and sufficiently large period of downloading for the sufficiently large amount of data as per our need for the furthur study. This type of huge personal workload can be minimized if the required data is compressed. It is a challenging task to compress the pixels without affecting the quality of image by compression techniques. In modern technology, the computer has the capacity to store a large amount of data as per need of modern society. This problem is affected by low internet connection due to which it takes a large amount of time to download the considerable amount of data. It is remarkable to note that wavelet transform and the exapansion of signal by wavelet methods increase the speed of this process. The wavelet expansions of signals have sufficient applications and role in deducting disrupt in the functioning of signals. If a researcher is interested to download the image of a signal by the help of computer then computer perform this task by the wavelet transform matrix of the concerned signal. This procedure is completely depending on approximation coefficients of the wavelet expansions of the series of concerned signals. When a perticular information is generated by the help of wavelets then it can be converted into its image. This process is continued until the original image is reconstructed. Now a days, several steps of data compression can be perfomed by wavelet. The collection of finger print cards are performed by using the compression techniques as per need of FBI(Federal Beuro of Investigation). There is a need of larger storage space for this technique. Thus the researchers feel difficulties. This difficulty is increased in case of storage space and requirements of some informations. A number of earlier mentioned problems of compression of data can be solved by the help of wavelets like Haar wavelet, Maxican wavelets, Shannon wavelets etc.

Signal processing is an advanced technology for the processing of images using mathematical operations by using some forms of signal processing. In this process, input is an image, a series of images or video of frames and output of image processing is either an image or a set of characteristics in the form of parameters related to the image concerned. Thus, signal processing encompasses the fundamental theory, algorithm, transferring informations and applications.In this process, the informations are transfered in several different physical, symbolic or abstract formats which depends on the designing of the signals.

Mathematical, statistical, linguistic, computational representations are useful in modelling, synthesis, analysis, discovery as well as recovery and sensing and learning of technology and engineering of signal processing.

In general, image processing is concerned to digital image processing but in the major, there are possibilities of optical as well as analog image processing.

There are important role of computer graphics and computer vision in image processing. In computer graphics, images are made by the help of following techniques: 
(1) Physical models of objects, enviorments and lightening from natural resources (scenes) which are possible in most animated movies.

In case of computer vision, high-level image processing is generally considered. In this process, a machine, a computer software intends to decipher the physical aspects of an image are helpful to study the properties of images consist of videos or 3D full body magnetic resonance scans.

In advance science and technology, generally images gain very wide scopes. In case of scientific visualization, it includes micro array data.

Ihe classical numerical analysis techniques contain the principles of signal processing and digital control systems include the important concepts of digitalization on digital refinements of related methods. The objectives of this paper are mentioned below :

(i) The expansion of signals(as a function of single variable) using Haar scaling functions and Haar wavelet to demostrate the imortance of wavelet exapansion.

(ii) The expansion of signal(as a function of of two variables) using double Haar scaling function and double Haar wavelet function and its verification for the correctness of introduced expansions by several examples.

(iii) The expansion of signal as a function $f(t)$ of single variable belonging to Lip $\alpha$ class $0<\alpha \leq 1$

(iv) To generate the concept of the signals as a function $f\left(t_{1}, t_{2}\right)$ of class $\operatorname{Lip}(\alpha, \beta)$ of two variables and to discuss the importance of these signals

(v) To investigate the nature of above mentioned signals(or functions) and to study in detail the performance of images of these signals under specific conditions

(vi) To decompose original signal and image and reconstruct original signal from decomposed signal.

\section{DEFINITIONS AND PRELIMINARIES}

\subsection{Signal}

A signal is variation of physical (measurable) quantity over time. Signal is composed of number of fundamental waves like sine

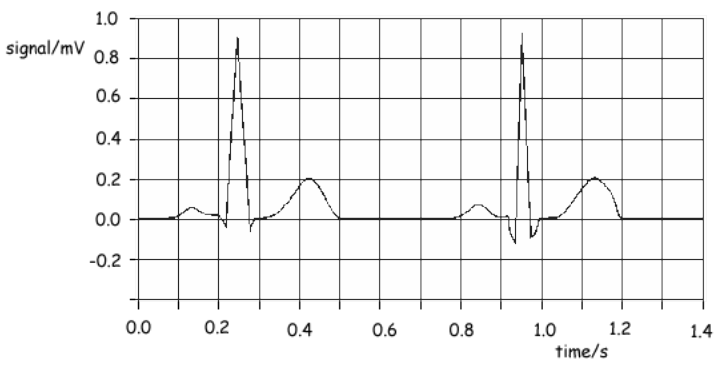

Fig. 1. Graph of ECG

waves, cosine waves etc with different frequencies, amplitudes and phases. The time of appearance of concerned waves are different within the time interval of informations received by signals. Signal can be of two types i.e. Stationary and Non Stationary (transient).

Stationary signal consists of waves having different frequencies and amplitudes. Times of presence of all component waves within the time interval (in which signal exists) are same and present in the entire time interval (in which signal exists). Phases of these waves are also same i.e. these waves start at phase zero.

Non Stationary (transient) signal is composed of waves having different frequencies, amplitudes, phases and with different time of appearance within time interval (in which signal exists) i.e. component waves usually appear for short amount of time (within time interval taken into consideration for the signal) and may start with different phases.

\section{Time and Space tradeoffs}

Data needs to be transformed into other form provided that it may be constructed back to its original form in order to minimize amount of time (transfer time when data is transferred over network) and storage space.

\section{Decomposition of a Signal}

Taking all possible combination of frequencies, phases and time of presence within time interval considered of fundamental waves and calculating their amplitudes w.r.t. the signal.

\section{Reconstruction of the signal}

Summing up all the waves according to their time of presence.

\section{Fourier Transform}

Set $\left\{e^{i \omega t}=\cos (\omega t)-i \sin (\omega t)\right\}$ forms orthonormal basis in $L^{2}(\mathbb{R})$ where

$<f, e^{i \omega t}>$ is amplitude of sinusoidal wave for fixed $\omega$.

Signal is decomposed by calculating amplitudes of sinusoidal waves for all $\omega$ w.r.t. $f(t)$ i.e. calculate $<f, e^{i \omega t}>$ for all $\omega$ and is reconstructed by taking linear combination of all waves i.e. $\sum_{-\infty}^{\infty}<f, e^{i \omega t}>e^{i \omega t}$.In this way, Fourier transform converts signal in time domain into signal in frequency domain. Stationary signal can be best decomposed and reconstructed using Fourier transform. But for decomposition of non stationary signal, an orthonormal basis is needed, which contains waves of all possible combination of different frequencies as well as different phases and different time of presence within the time interval.

\section{Role of Wavelet in Signal Processing}

In 1982, Morlet first time introduced the idea of wavelets as a family of functions constructed from translations and dilations of a single function called mother wavelet. later Meyer and Mallat recognized that construction of different wavelet bases can be realized by the so called multiresolution analysis. The fundamental idea of multiresolution analysis is to represent a function as a limit of successive approximations, each of which is a smoother version of the original function. The successive approximation corresponds to different resolution, which leads to the name multiresolution analysis as a formal approach to constructing orthonormal wavelet bases using resonable rules and methods.

\subsection{Multiresolution Analysis ( MRA)}

Mutiresolution analyses (MRA) with father wavelet or scaling function $\varphi$ consists of a sequence of closed subspaces $\left\{V_{j}\right\}_{j \in \mathbb{Z}}$ of $L^{2}(\mathbb{R})$ satisfying the following properties.

Monotonicity

The sequence is increasing. $V_{j} \subseteq V_{j+1}$ for all $j \in \mathbb{Z}$.

\section{Existence of Scaling Function}


There exist a function $\varphi \in V_{0}$ such that $\varphi_{0, n}=\{\varphi(t-n), n \in \mathbb{Z}\}$ is an orthonormal basis for all $V_{0}$,

i.e. $\|f\|_{2}^{2}=\int_{-\infty}^{\infty}|f|^{2} d t=\sum\left|<f, \varphi_{0, n}>\right|^{2}$ for all $f \in V_{0}$.

\section{Dilation Property}

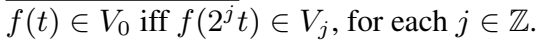

\section{Trivial Intersection Property}

$\cap_{j \in \mathbb{Z}} V_{j}=\{0\}$

\section{Density}

$\overline{\overline{\cup_{j \in \mathbb{Z}} V_{j}}}=L^{2}(\mathbb{R})$

\subsection{Haar Scaling Function and Father Wavelet in single variable}

A function $\varphi \in L^{2}(\mathbb{R})$ over the interval $[0,1)$ is defined as follows

$$
\begin{gathered}
\varphi=\chi_{[0,1)} \\
\text { or } \\
\varphi(t)= \begin{cases}1 & \text { if } 0 \leq t<1, \\
0 & \text { otherwise }\end{cases}
\end{gathered}
$$

is called Haar scaling function in one variable.

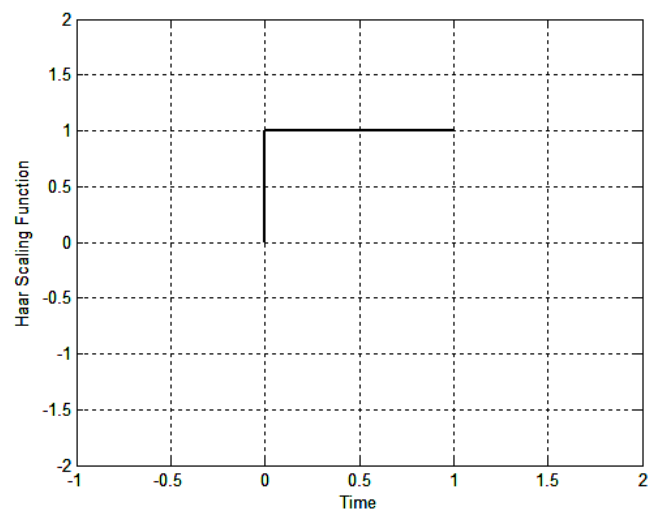

Fig. 2. Graph of one dimentional Haar scaling function $(\varphi)$

Dilated and translated version $\varphi_{j, k}$ is defined as follows :

$$
\varphi_{j, k}(t)=2^{j / 2} \varphi\left(2^{j} t-k\right)
$$

where

$$
\varphi\left(2^{j} t-k\right)= \begin{cases}1 & \text { if } k / 2^{j} \leq t<(k+1) / 2^{j} \\ 0 & \text { otherwise }\end{cases}
$$

is called a system of Haar scaling functions.

$\varphi$ satisfies the following properties:

(i) $\varphi \in L^{2}(\mathbb{R})$.

(ii) Scaling function $\varphi_{j, k}(t)$ are supported on a dyadic interval.

i.e. $I_{j, k}=\left[k / 2^{j},(k+1) / 2^{j}\right)$ for each $j, k \in \mathbb{Z}$

(iii) $V_{j}=\operatorname{span}\left\{\varphi_{j, k}, j, k \in \mathbb{Z}\right\}$.

$\left\{V_{j}\right\}_{j=-\infty}^{\infty}$ is a Multiresolution Analysis of $L^{2}(\mathbb{R})$

(iv) $\int_{-\infty}^{\infty} \varphi(t) d t \neq 0$ (v) $\varphi(t)=\sum_{n=-\infty}^{n=\infty} c_{n} \varphi(2 t-n) ; n \in \mathbb{Z}$

where $c_{n}=<\varphi(t), \varphi(2 t-n)>$

\subsection{Haar Scaling Function and Father Wavelet in two variable}

Let $\mathbb{R}$ be the set of real numbers. A function $\Phi:[0,1) \times[0,1) \rightarrow \mathbb{R}$ defined by

$$
\Phi\left(t_{1}, t_{2}\right)=\varphi\left(t_{1}\right) \varphi\left(t_{2}\right)
$$

is called a Haar Scaling function in two variable $t_{1}$ and $t_{2}$

$$
\begin{aligned}
\Phi_{j, k ; j^{\prime}, k^{\prime}}\left(t_{1}, t_{2}\right) & =\varphi_{j, k}\left(t_{1}\right) \varphi_{j^{\prime}, k^{\prime}}\left(t_{2}\right) \\
& =2^{j / 2} 2^{j^{\prime} / 2} \varphi\left(2^{j} t_{1}-k\right) \varphi\left(2^{j^{\prime}} t_{2}-k^{\prime}\right)
\end{aligned}
$$

\subsection{Haar Wavelet Function}

A function $\psi \in L^{2}(\mathbb{R})$ over the interval $[0,1)$ is defined as follows:

$$
\begin{gathered}
\psi=\chi_{[0,1 / 2)}-\chi_{[1 / 2,1)} \\
\text { or } \\
\psi= \begin{cases}1 & \text { if } 0 \leq t<1 / 2, \\
-1 & \text { if } 1 / 2 \leq t<1, \\
0 & \text { otherwise }\end{cases}
\end{gathered}
$$

is called a Haar wavelet function.

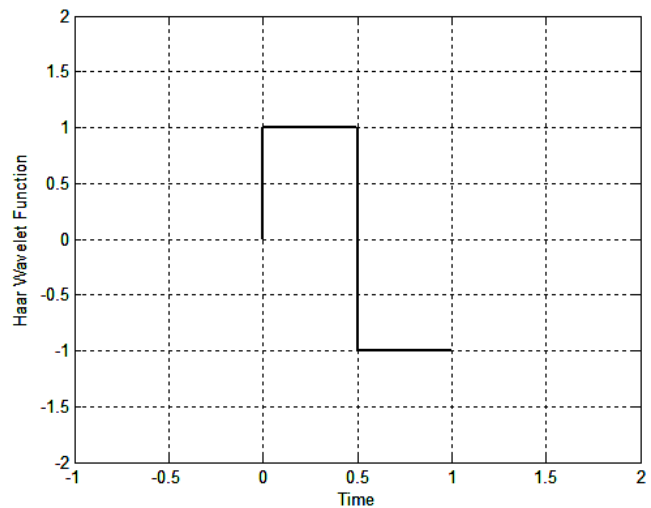

Fig. 3. Graph of one dimentional Haar wavelet function $(\psi)$

Dilated and translated version $\psi_{j, k}$ is defined by :

$$
\begin{aligned}
\psi_{j, k}(t) & =2^{j / 2} \psi\left(2^{j} t-k\right) \\
& = \begin{cases}2^{j / 2} & \text { if } k / 2^{j} \leq t<(k+1 / 2) / 2^{j}, \\
-2^{j / 2} & \text { if }(k+1 / 2) / 2^{j} \leq t<(k+1) / 2^{j}, \\
0 & \text { otherwise }\end{cases}
\end{aligned}
$$

Haar wavelet $\psi$ satisfies following properties:

(i) $\psi \in L^{2}(\mathbb{R})$.

(ii) Wavelet function $\psi_{j, k}(t)$ are supported on a dyadic interval. i.e. $I_{j, k}=\left[k / 2^{j},(k+1) / 2^{j}\right)$ for each $j, k \in \mathbb{Z}$

(iii) For each j, $W_{j}=\operatorname{clos}_{L^{2}(\mathbb{R})}<\psi_{j, k}: k \in \mathbb{Z}>$, $L^{2}(\mathbb{R})=\sum_{j \in \mathbb{Z}} W_{j}$ 
(iv) $\psi$ satisfies admissibility condition $\left(C_{\psi}\right)$ defined as follows:

$$
C_{\psi}=\frac{\int_{-\infty}^{\infty}|\hat{\psi}(\omega)|^{2} d \omega}{|\omega|}<0 \Rightarrow \psi(0)=0 \Leftrightarrow \int_{-\infty}^{\infty} \psi(t) d t=0
$$

(v) $\psi(t)=\sum_{n=-\infty}^{n=\infty}(-1)^{n} c_{n+1} \varphi(2 t+n) ; n \in \mathbb{Z}$

where $c_{n+1}=<\psi(t), \varphi(2 t+n)>$

\subsection{Haar Wavelet Function in two variable}

A function $\Psi:[0,1) \times[0,1) \rightarrow \mathbb{R}$ is defined by

$$
\left.\Psi\left(t_{1}, t_{2}\right)=\psi\left(t_{1}\right) \psi_{(} t_{2}\right)
$$

is called Haar wavelet in two variables $t_{1}$ and $t_{2}$, where $\psi(t)=\chi_{[0,1 / 2)}(t)-\chi_{[1 / 2,1)}(t)$.

$$
\begin{aligned}
\Psi\left(t_{1}, t_{2}\right)= & \left(\chi_{[0,1 / 2)}\left(t_{1}\right)-\chi_{[1 / 2,1)}\left(t_{1}\right)\right)\left(\chi_{[0,1 / 2)}\left(t_{2}\right)\right. \\
& \left.-\chi_{[1 / 2,1)}\left(t_{2}\right)\right) \\
= & \chi_{[0,1 / 2)}\left(t_{1}\right) \chi_{[0,1 / 2)}\left(t_{2}\right)-\chi_{[0,1 / 2)}\left(t_{1}\right) \chi_{[1 / 2,1)}\left(t_{2}\right) \\
- & \chi_{[1 / 2,1)}\left(t_{1}\right) \chi_{[0,1 / 2)}\left(t_{2}\right)+\chi_{[1 / 2,1)}\left(t_{1}\right) \chi_{[1 / 2,1)}\left(t_{2}\right) \\
= & \left\{\begin{array}{rl}
1 & 0 \leq t_{1}, t_{2}<1 / 2 \\
-1 & 0 \leq t_{1}<1 / 2,1 / 2 \leq t_{2}<1 \\
-1 & 1 / 2 \leq t_{1}<1,0 \leq t_{2}<1 / 2 \\
1 & 1 / 2 \leq t_{1}, t_{2}<1 \\
0 & \text { otherwise }
\end{array}\right.
\end{aligned}
$$

\section{SINGLE HAAR WAVELET SERIES}

3.1 Haar Wavelet Series for a signal $f \in L^{2}(\mathbb{R})$ over the interval $[0,1)$ at resolution $m$.

Wavelet series of a signal $f \in L^{2}(\mathbb{R})$ using Haar scaling function $\phi$ and Haar wavelet function $\psi$ is written as

$$
f(t)=\sum_{n=0}^{2^{m}-1} c_{m, n} \varphi_{m, n}(t)+\sum_{j=m}^{\infty} \sum_{k=0}^{2^{j}-1} d_{j, k} \psi_{j, k}(t)
$$

where

$$
c_{m, n}=<f, \varphi_{m, n}>, d_{j, k}=<f, \psi_{j, k}>.
$$

For $m=0$ the series (1) reduces to,

$$
f(t)=c_{0,0} \varphi_{0,0}(t)+\sum_{j=0}^{\infty} \sum_{k=0}^{2^{j}-1} d_{j, k} \psi_{j, k}(t)
$$

\begin{tabular}{|c|c|c|}
\hline Cases & {$[0,3 / 4) \cap\left[k / 2^{j},(k+1) / 2^{j}\right)$} & Values of $j$ and $k$ satisfied \\
\hline (1). & $\begin{array}{c}{\left[k / 2^{j},(k+1) / 2^{j}\right)} \\
\text { overlapes }[0,3 / 4)\end{array}$ & $j=k=0$ \\
\hline (2). & $\begin{array}{c}{[0,3 / 4) \text { intersects }} \\
{\left[k / 2^{j},(k+1) / 2^{j}\right)}\end{array}$ & $j=k=1$ \\
\hline (3). & $\begin{array}{l}{[0,3 / 4) \text { overlapes }} \\
{\left[k / 2^{j},(k+1) / 2^{j}\right)}\end{array}$ & $\begin{array}{c}j \geq 1, k=0 \text { and } \\
j \geq 2,1 \leq k \leq\left(3 * 2^{j-2}-1\right)\end{array}$ \\
\hline
\end{tabular}

For $f(t)=\chi_{[0,3 / 4)}(t)$, the value of $c_{m, n}$ and $d_{j, k}$ are calculated considering table 1

By rewriting equation (2) w.r.t.the conditions mentioned in the table $(1)$, we can say that,

Any signal $f \in L^{2}(\mathbb{R})$ over the interval $[0,3 / 4)$ can be expanded at resolution $m=0$ in single Haar wavelet series as

$$
\begin{aligned}
f(t)= & c_{0,0} \varphi_{0,0}(t)+d_{0,0} \psi_{0,0}(t)+d_{1,1} \psi_{1,1}(t)+\sum_{j=1}^{\infty} d_{j, 0} \psi_{j, 0}(t) \\
& +\sum_{j=2}^{\infty} \sum_{k=1}^{3 * 2^{j-2}-1} d_{j, k} \psi_{j, k}(t)
\end{aligned}
$$

Table 1.

\section{DOUBLE HAAR WAVELET SERIES}

\subsection{Double Haar Wavelet Series for a signal} $f \in L^{2}(\mathbb{R} \times \mathbb{R})$ in the region $[0,1) \times[0,1)$ at resolutions $m_{1}=m$ and $m_{2}=m^{\prime}$.

A signal $f:[0,1) \times[0,1) \rightarrow \mathbb{R}$ is expressed as double wavelet series using doublw Haar scaling function $\Phi$ and Haar mother wavelet $\Psi$ in the following form:

$$
\begin{aligned}
f\left(t_{1}, t_{2}\right) & =\sum_{n=0}^{2^{m}-1} \sum_{n^{\prime}=0}^{2^{m^{\prime}}-1} c_{m, n ; m^{\prime}, n^{\prime}} \varphi_{m, n ; m^{\prime}, n^{\prime}}\left(t_{1}, t_{2}\right) \\
& +\sum_{n=0}^{2^{m}-1} \sum_{j^{\prime}=m^{\prime}}^{\infty} \sum_{k^{\prime}=0}^{2^{j^{\prime}}-1} c_{m, n ; j^{\prime}, k^{\prime}}^{\prime} \varphi_{m, n ; j^{\prime}, k^{\prime}}^{\prime}\left(t_{1}, t_{2}\right) \\
& +\sum_{j=m}^{\infty} \sum_{k=0}^{2^{j}-1} \sum_{n^{\prime}=0}^{2^{\prime}-1} d_{j, k ; m^{\prime}, n^{\prime}}^{\prime} \psi_{j, k ; m^{\prime}, n^{\prime}}^{\prime}\left(t_{1}, t_{2}\right) \\
& +\sum_{j=m}^{\infty} \sum_{k=0}^{2^{j}-1} \sum_{j^{\prime}=m^{\prime}}^{\infty} \sum_{k^{\prime}=0}^{2^{j^{\prime}-1}} d_{j, k ; j^{\prime}, k^{\prime}} \psi_{j, k ; j^{\prime}, k^{\prime}}\left(t_{1}, t_{2}\right)
\end{aligned}
$$

where

$$
\begin{gathered}
c_{m, n ; m^{\prime}, n^{\prime}}=<f, \varphi_{m, n ; m^{\prime}, n^{\prime}}>, \\
c_{m, n ; j^{\prime}, k^{\prime}}^{\prime}=<f, \varphi_{m, n ; j^{\prime}, k^{\prime}}^{\prime}>, \\
d_{j, k ; m^{\prime}, n^{\prime}}^{\prime}=<f, \psi_{j, k ; m^{\prime}, n^{\prime}}^{\prime}>, \\
d_{j, k ; j^{\prime}, k^{\prime}}=<f, \psi_{j, k ; j^{\prime}, k^{\prime}}>, \\
\varphi_{m, n ; m^{\prime}, n^{\prime}}\left(t_{1}, t_{2}\right)=\varphi_{m, n}\left(t_{1}\right) \varphi_{m^{\prime}, n^{\prime}}\left(t_{2}\right), \\
\varphi_{m, n ; j^{\prime}, k^{\prime}}^{\prime}\left(t_{1}, t_{2}\right)=\varphi_{m, n}\left(t_{1}\right) \psi_{j^{\prime}, k^{\prime}}\left(t_{2}\right), \\
\psi_{j, k ; m^{\prime}, n^{\prime}}^{\prime}\left(t_{1}, t_{2}\right)=\psi_{j, k}\left(t_{1}\right) \varphi_{m^{\prime}, n^{\prime}}\left(t_{2}\right), \\
\psi_{j, k ; j^{\prime}, k^{\prime}}\left(t_{1}, t_{2}\right)=\psi_{j, k}\left(t_{1}\right) \psi_{j^{\prime}, k^{\prime}}\left(t_{2}\right) .
\end{gathered}
$$




\subsection{Double Haar Wavelet Series for a signal} $f \in L^{2}(\mathbb{R} \times \mathbb{R})$ in the region $[0,3 / 4) \times[0,3 / 4)$ at resolutions $m_{1}=m_{2}=0$.

For $f\left(t_{1}, t_{2}\right)=\chi_{[0,3 / 4)}\left(t_{1}\right) \chi_{[0,3 / 4)}\left(t_{2}\right), m=m^{\prime}=0$, the series 4 reduces to

$$
\begin{aligned}
& f\left(t_{1}, t_{2}\right)=c_{0,0 ; 0,0} \varphi_{0,0 ; 0,0}\left(t_{1}, t_{2}\right)+c_{0,0 ; 0,0}^{\prime} \varphi_{0,0 ; 0,0}^{\prime}\left(t_{1}, t_{2}\right) \\
& +c_{0,0 ; 1,1}^{\prime} \varphi_{0,0 ; 1,1}^{\prime}\left(t_{1}, t_{2}\right)+\sum_{j^{\prime}=1}^{\infty} c_{0,0 ; j^{\prime}, 0}^{\prime} \varphi_{0,0 ; j^{\prime}, 0}^{\prime}\left(t_{1}, t_{2}\right) \\
& +\sum_{j^{\prime}=2}^{\infty} \sum_{k^{\prime}=1}^{3 * j^{j^{\prime}-2}-1} c_{0,0 ; j^{\prime}, k^{\prime}}^{\prime} \varphi_{0,0 ; j^{\prime}, k^{\prime}}^{\prime}\left(t_{1}, t_{2}\right) \\
& +d_{0,0 ; 0,0}^{\prime} \psi_{0,0 ; 0,0}^{\prime}\left(t_{1}, t_{2}\right)+d_{0,0 ; 0,0} \psi_{0,0 ; 0,0}\left(t_{1}, t_{2}\right) \\
& +d_{0,0 ; 1,1} \psi_{0,0 ; 1,1}\left(t_{1}, t_{2}\right)+\sum_{j^{\prime}=1}^{\infty} d_{0,0 ; j^{\prime}, 0} \psi_{0,0 ; j^{\prime}, 0}\left(t_{1}, t_{2}\right) \\
& +\sum_{j^{\prime}=2}^{\infty} \sum_{k^{\prime}=1}^{3 * j^{j^{\prime}-2}-1} d_{0,0 ; j^{\prime}, k^{\prime}} \psi_{0,0 ; j^{\prime}, k^{\prime}}\left(t_{1}, t_{2}\right) \\
& +d_{1,1 ; 0,0}^{\prime} \psi_{1,1 ; 0,0}^{\prime}\left(t_{1}, t_{2}\right)+d_{1,1 ; 0,0} \psi_{1,1 ; 0,0}\left(t_{1}, t_{2}\right) \\
& +d_{1,1 ; 1,1} \psi_{1,1 ; 1,1}\left(t_{1}, t_{2}\right)+\sum_{j^{\prime}=1}^{\infty} d_{1,1 ; j^{\prime}, 0} \psi_{1,1 ; j^{\prime}, 0}\left(t_{1}, t_{2}\right) \\
& +\sum_{j^{\prime}=2}^{\infty} \sum_{k^{\prime}=1}^{3 * 2^{j^{\prime}-2}-1} d_{1,1 ; j^{\prime} k^{\prime}} \psi_{1,1 ; j^{\prime}, k^{\prime}}\left(t_{1}, t_{2}\right) \\
& +\sum_{j=1}^{\infty} d_{j, 0 ; 0,0}^{\prime} \psi_{j, 0 ; 0,0}^{\prime}\left(t_{1}, t_{2}\right) \\
& +\sum_{j=1}^{\infty} d_{j, 0 ; 0,0} \psi_{j, 0 ; 0,0}\left(t_{1}, t_{2}\right)+\sum_{j=1}^{\infty} d_{j, 0 ; 1,1} \psi_{j, 0 ; 1,1}\left(t_{1}, t_{2}\right) \\
& +\sum_{j=1}^{\infty} \sum_{j^{\prime}=1}^{\infty} d_{j, 0 ; j^{\prime}, 0} \psi_{j, 0 ; j^{\prime}, 0}\left(t_{1}, t_{2}\right) \\
& +\sum_{j=1}^{\infty} \sum_{j^{\prime}=2}^{\infty} \sum_{k^{\prime}=1}^{3 * 2^{j^{\prime}-2}-1} d_{j, 0 ; j^{\prime}, k^{\prime}} \psi_{j, 0 ; j^{\prime}, k^{\prime}}\left(t_{1}, t_{2}\right) \\
& +\sum_{j=2}^{\infty} \sum_{k=1}^{3 * 2^{j-2}-1} d_{j, k ; 0,0}^{\prime} \psi_{j, k ; 0,0}^{\prime}\left(t_{1}, t_{2}\right) \\
& +\sum_{j=2}^{\infty} \sum_{k=1}^{3 * 2^{j-2}-1} d_{j, k ; 0,0} \psi_{j, k ; 0,0}\left(t_{1}, t_{2}\right) \\
& +\sum_{j=2}^{\infty} \sum_{k=1}^{3 * 2^{j-2}-1} d_{j, k ; 1,1} \psi_{j, k ; 1,1}\left(t_{1}, t_{2}\right) \\
& +\sum_{j=2}^{\infty} \sum_{k=1}^{3 * 2^{j-2}-1} \sum_{j^{\prime}=1}^{\infty} d_{j, k ; j^{\prime}, 0} \psi_{j, k ; j^{\prime}, 0}\left(t_{1}, t_{2}\right) \\
& +\sum_{j=2}^{\infty} \sum_{k=1}^{3 * 2^{j-2}} \sum_{j^{\prime}=2}^{\infty} \sum_{k^{\prime}=1}^{3 * 2^{j^{\prime}-2}-1} d_{j, k ; j^{\prime}, k^{\prime}} \psi_{j, k ; j^{\prime}, k^{\prime}}\left(t_{1}, t_{2}\right)
\end{aligned}
$$

\section{DECOMPOSITION AND RECONSTRUCTION OF A SIGNAL}

\subsection{Decomposition of signal}

The decomposition of a signal can be performed by the help of following steps:

(i) Needs to be discritized such that total number of discrete points will be in exponent of 2 and minimum number of discrete points will be $2^{(m+1)}$. i.e. space between two discrete points will be $(b-a) / 2^{r}$ if $2^{r}$ is total number of discrete points taken provided $r \geq(m+1)$

(ii) Construct Haar wavelet matrix of $2^{r} \times 2^{r}$ for resolution $m$.

(iii) Use following formulae to obtain decomposed signal

$[\text { DecomposedSignal }]_{1 \times 2^{r}}=[\text { OriginalSignal }]_{1 \times 2^{r}}$

* $[\text { HaarWaveletMatrix }]_{2^{r} \times 2^{r}}$

\subsection{Reconstruction of signal}

The reconstruction of a signal from decomposed signal is obtained by following formula:

$[\text { ReconstructedSignal }]_{1 \times 2^{r}}=[\text { DecomposedSignal }]_{1 \times 2^{r}}$

* $[\text { HaarWaveletMatrix }]_{2^{r} \times 2^{r}}^{-1}$

\section{LIPSCHITZ CLASS}

\section{1 $\operatorname{Lip}_{\alpha}[a, b]$ Class}

A function $f \in \operatorname{Lip}_{\alpha}[a, b]$ for any $0<\alpha \leq 1$ if

$$
\begin{array}{r}
\left|f\left(t_{1}\right)-f\left(t_{2}\right)\right|=O\left(\left|\left(t_{1}-t_{2}\right)\right|^{\alpha}\right) \forall t_{1}, t_{2} \in[a, b], \\
(\text { Titchmarsh }[2], p .406)
\end{array}
$$

Define $f:[0,1] \rightarrow \mathbb{R}$ by $f(t)=t^{\alpha}, \quad \forall t \in[0,1), 0<\alpha \leq 1$

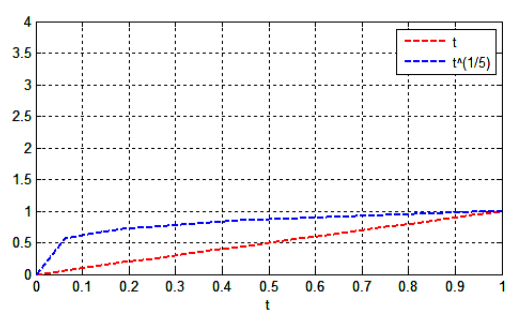

Fig. 4. Graph of signal $f_{1}(t)=t, t \in[0,1]$ and $f_{2}(t)=t^{\alpha}, t \in[0,1]$ for $\alpha=1 / 5$

\section{2 $\operatorname{Lip}_{\alpha, \beta}([a, b] \times[c, d])$ class}

A function $f$ of two variables $t_{1}$ and $t_{2}$ belongs to $\operatorname{Lip}_{(\alpha, \beta)}$ if $\left|f\left(t_{1}+u, t_{2}+v\right)-f\left(t_{1}, t_{2}\right)\right|=O\left(|u|^{\alpha}+|v|^{\beta}\right), \quad 0<\alpha, \beta \leq 1$. Define $f:[0,1] \times[0,1] \rightarrow \mathbb{R}$ by $f\left(t_{1}, t_{2}\right)=t_{1}^{\alpha}+t_{2}^{\alpha}, \forall\left(t_{1}, t_{2}\right) \in$ $[0,1] \times[0,1], 0<\alpha, \beta \leq 1$ 


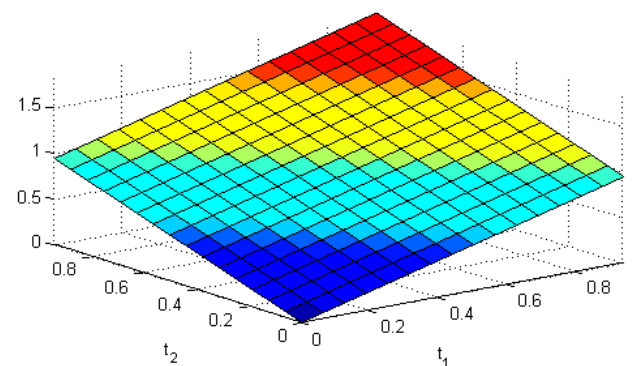

Fig. 5. Graph of signal $f_{1}\left(t_{1}, t_{2}\right)=t_{1}+t_{2},\left(t_{1}, t_{2}\right) \in[0,1] \times[0,1]$

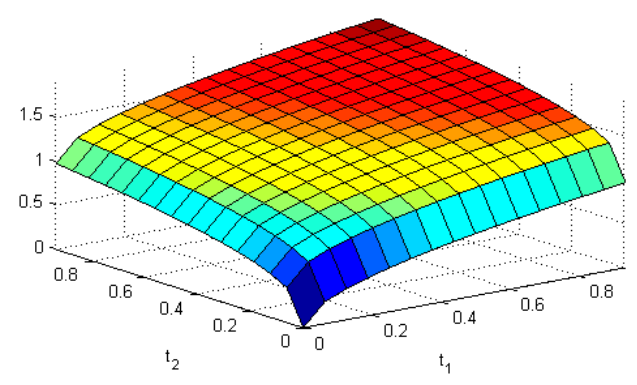

Fig. 6. Graph of signal $\left.f_{2}\left(t_{1}, t_{2}\right)=t_{1}^{\alpha}+t_{2}^{\alpha},\left(t_{1}, t_{2}\right) \in[0,1] \times[0,1]\right)$ for $\alpha=1 / 2, \beta=1 / 3$

\section{EXAMPLES}

7.1 Expansion of signal $f(t)=t, t \in[0,1)$ at resolution $m=0$.

A signal $f:[0,1) \rightarrow \mathbb{R}$ is defined by $f(t)=t, t \in[0,1)$.

The wavelet series of $f$ using $\phi$ and $\psi$ is given by

$$
f(t)=c_{0,0} \varphi_{0,0}(t)+\sum_{j=0}^{\infty} \sum_{k=0}^{2^{j}-1} d_{j, k} \psi_{j, k}(t)
$$

To evaluate $c_{m, n}$

$$
\begin{aligned}
c_{m, n} & =\left\langle f, \varphi_{m, n}>=\int_{-\infty}^{\infty} f(t) \varphi_{m, n}(t) d t\right. \\
& =\int_{n / 2^{m}}^{(n+1) / 2^{m}} t * 2^{m / 2} d t=2^{m / 2} \int_{n / 2^{m}}^{(n+1) / 2^{m}} t d t \\
& =\frac{1}{2^{3 m / 2}}\left(n+\frac{1}{2}\right)
\end{aligned}
$$

To evaluate $d_{j, k}$

$$
\begin{aligned}
d_{j, k} & =\left\langle f, \psi_{j, k}>=\int_{-\infty}^{\infty} f(t) \psi_{j, k}(t) d t\right. \\
& =\int_{k / 2^{j}}^{(k+1) / 2^{j}} t * \psi_{j, k}(t) d t \\
& =\int_{k / 2^{j}}^{(k+1 / 2) / 2^{j}} t * 2^{j / 2} d t-\int_{(k+1 / 2) / 2^{j}}^{(k+1) / 2^{j}} t * 2^{j / 2} d t \\
& =2^{j / 2}\left(\int_{k / 2^{j}}^{(k+1 / 2) / 2^{j}} t d t-\int_{(k+1 / 2) / 2^{j}}^{(k+1) / 2^{j}} t d t\right) \\
& =\frac{1}{2} \frac{1}{2^{3 j / 2}}\left\{2\left(k+\frac{1}{2}\right)^{2}-k^{2}-(k+1)^{2}\right\} \\
& =-\frac{1}{4} \frac{1}{2^{3 j / 2}}
\end{aligned}
$$

From equations 6 to 8

$$
f(t)=t=\frac{1}{2} \varphi_{0,0}(t)+\sum_{j=0}^{\infty} \sum_{k=0}^{2^{j}-1} \frac{(-1)}{4} \frac{1}{2^{3 j / 2}} \psi_{j, k}(t)
$$

Then,

$$
\begin{aligned}
\|f\|_{2}^{2} & =\left(\frac{1}{2}\right)^{2}\left\|\varphi_{0,0}\right\|_{2}^{2} \\
& +\sum_{j=0}^{\infty} \sum_{k=0}^{2^{j}-1}\left(\frac{-1}{4}\right)^{2}\left(\frac{1}{2^{3 j / 2}}\right)^{2}\left\|\psi_{j, k}\right\|_{2}^{2} \\
& =\frac{1}{4}\left\|\varphi_{0,0}\right\|_{2}^{2}+\frac{1}{16} \sum_{j=0}^{\infty} \sum_{k=0}^{2^{j}-1} \frac{1}{2^{3 j}}\left\|\psi_{j, k}\right\|_{2}^{2}
\end{aligned}
$$

$$
\begin{aligned}
\left\|\varphi_{m, n}\right\|_{2}^{2} & =\int_{-\infty}^{\infty}\left|\varphi_{m, n}(t)\right|^{2} d t=\int_{n / 2^{m}}^{(n+1) / 2^{m}}\left(2^{m / 2}\right)^{2} d t \\
& =2^{m} \int_{n / 2^{m}}^{(n+1) / 2^{m}} d t=1
\end{aligned}
$$

$$
\begin{aligned}
\left\|\psi_{j, k}\right\|_{2}^{2} & =\int_{-\infty}^{\infty}\left|\psi_{j, k}(t)\right|^{2} d t=\int_{k / 2^{j}}^{(k+1) / 2^{j}}\left|\psi_{j, k}(t)\right|^{2} d t \\
& =\int_{k / 2^{j}}^{(k+1 / 2) / 2^{j}}\left(2^{j / 2}\right)^{2} d t+\int_{(k+1 / 2) / 2^{j}}^{(k+1) / 2^{j}}\left(-2^{j / 2}\right)^{2} d t \\
& =2^{j}\left(\int_{k / 2^{j}}^{(k+1 / 2) / 2^{j}} d t+\int_{(k+1 / 2) / 2^{j}}^{(k+1) / 2^{j}} d t\right) \\
& =2^{j}\left\{\left(k+\frac{1}{2}\right) \frac{1}{2^{j}}-\frac{k}{2^{j}}+\frac{(k+1)}{2^{j}}-\left(k+\frac{1}{2}\right) \frac{1}{2^{j}}\right\} \\
& =1 \\
& \|f\|_{2}^{2}=\int_{-\infty}^{\infty}|f(t)|^{2} d t=\int_{0}^{1} t^{2} d t=\frac{1}{3} \\
& \frac{1}{4}\left\|\varphi_{0,0}\right\|_{2}^{2}+\frac{1}{16} \sum_{j=0}^{\infty} \sum_{k=0}^{2^{j}-1} \frac{1}{2^{3 j}}\left\|\psi_{j, k}\right\|_{2}^{2}=\frac{1}{4}
\end{aligned}
$$




$$
\begin{aligned}
& +\frac{1}{16} \sum_{j=0}^{\infty} \sum_{k=0}^{2^{j}-1} \frac{1}{2^{3 j}}=\frac{1}{4}+\frac{1}{16} \sum_{j=0}^{\infty} \frac{1}{2^{3 j}}\left(\sum_{k=0}^{2^{j}-1} 1\right) \\
& =\frac{1}{4}+\frac{1}{16}\left(\sum_{j=0}^{\infty} \frac{1}{2^{2 j}}\right)=\frac{1}{4}+\frac{1}{16}\left\{\frac{1}{1-1 / 4}\right\}=\frac{1}{3}
\end{aligned}
$$

The wavelet expansion 6 is verified.

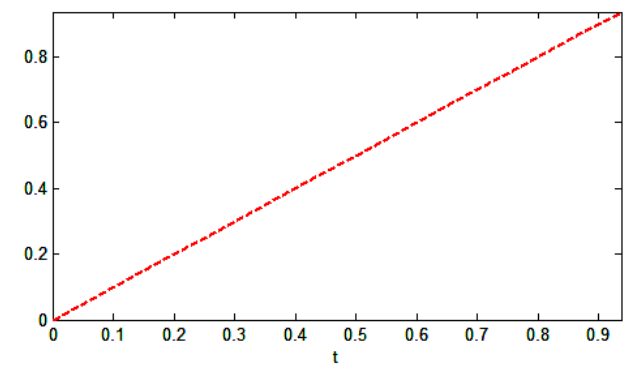

Fig. 7. Graph of signal $f(t)=t, t \in[0,1)$ with number of discrete points $=16$.

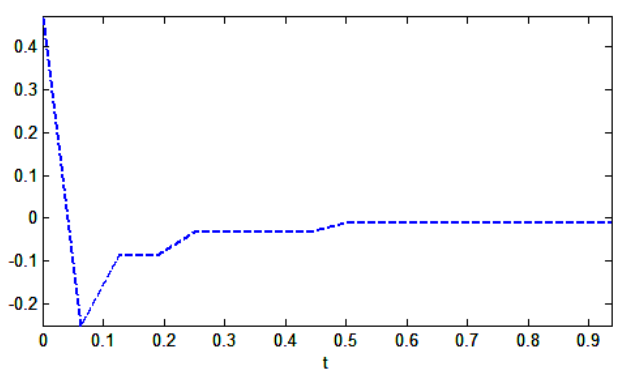

Fig. 8. Graph of decomposed signal $f(t)=t, t \in[0,1)$ with number of discrete points $=16$ at resolution $m=0$ using Haar wavelet matrix.

7.2 Expansion of signal $f(t)=t, t \in[0,1)$ at resolution $m=1$.

$$
\begin{gathered}
f(t)=\sum_{n=0}^{1} c_{1, n} \varphi_{1, n}(t)+\sum_{j=1}^{\infty} \sum_{k=0}^{2^{j}-1} d_{j, k} \psi_{j, k}(t) \\
c_{1, n}=\frac{1}{2^{3 / 2}}\left(n+\frac{1}{2}\right)
\end{gathered}
$$$$
f(t)=\sum_{n=0}^{1} \frac{1}{2^{3 / 2}}\left(n+\frac{1}{2}\right) \varphi_{1, n}(t)+\sum_{j=1}^{\infty} \sum_{k=0}^{2^{j}-1} \frac{(-1)}{4} \frac{1}{2^{3 j / 2}} \psi_{j, k}(t)
$$

taking square of norm of the equation both sides,

$$
\begin{aligned}
\|f\|_{2}^{2} & =\sum_{n=0}^{1}\left(\frac{1}{2^{3 / 2}}\right)^{2}\left(n+\frac{1}{2}\right)^{2}\left\|\varphi_{1, n}\right\|_{2}^{2} \\
& +\sum_{j=1}^{\infty} \sum_{k=0}^{2^{j}-1}\left(\frac{-1}{4}\right)^{2}\left(\frac{1}{2^{3 j / 2}}\right)^{2}\left\|\psi_{j, k}\right\|_{2}^{2} \\
& =\frac{1}{2^{3}} \sum_{n=0}^{1}\left(n+\frac{1}{2}\right)^{2}\left\|\varphi_{1, n}\right\|_{2}^{2} \\
& +\frac{1}{16} \sum_{j=1}^{\infty} \sum_{k=0}^{2^{j}-1} \frac{1}{2^{3 j}}\left\|\psi_{j, k}\right\|_{2}^{2}
\end{aligned}
$$

taking R.H.S of equation 10

$$
\begin{aligned}
\text { R.H.S } & =\frac{1}{2^{3}} \sum_{n=0}^{1}\left(n+\frac{1}{2}\right)^{2}\left\|\varphi_{1, n}\right\|_{2}^{2} \\
& +\frac{1}{16} \sum_{j=1}^{\infty} \sum_{k=0}^{2^{j}-1} \frac{1}{2^{3 j}}\left\|\psi_{j, k}\right\|_{2}^{2} \\
& =\frac{1}{2^{3}} \sum_{n=0}^{1}\left(n+\frac{1}{2}\right)^{2}+\frac{1}{16} \sum_{j=1}^{\infty} \sum_{k=0}^{2^{j}-1} \frac{1}{2^{3 j}} \\
& =\frac{1}{8}\left(\frac{1}{4}+\frac{9}{4}\right)+\frac{1}{16} \sum_{j=1}^{\infty} \frac{1}{2^{3 j}}\left(\sum_{k=0}^{2^{j}-1} 1\right) \\
& =\frac{5}{16}+\frac{1}{16}\left(\sum_{j=1}^{\infty} \frac{1}{2^{2 j}}\right) \\
& =\frac{5}{16}+\frac{1}{16}\left(\frac{1 / 4}{1-1 / 4}\right)=\frac{1}{3}
\end{aligned}
$$

Therefore 10 is satisfied.

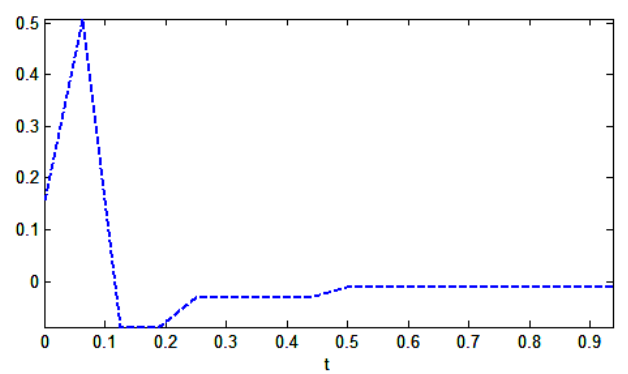

Fig. 9. Graph of decomposed signal $f(t)=t, t \in[0,1)$ with number of discrete points $=16$ at resolution $m=1$ using Haar wavelet matrix.

7.3 Expansion of signal $f(t)=t, t \in[0,1)$ at resolution $m$.

$$
f(t)=\sum_{n=0}^{2^{m}-1} c_{m, n} \varphi_{m, n}(t)+\sum_{j=m}^{\infty} \sum_{k=0}^{2^{j}-1} d_{j, k} \psi_{j, k}(t)
$$




$$
\begin{aligned}
& =\sum_{n=0}^{2^{m}-1} \frac{1}{2^{3 m / 2}}\left(n+\frac{1}{2}\right) \varphi_{m, n}(t) \\
& +\sum_{j=m}^{\infty} \sum_{k=0}^{2^{j}-1} \frac{(-1)}{4} \frac{1}{2^{3 j / 2}} \psi_{j, k}(t) \\
\|f\|_{2}^{2} & =\frac{1}{2^{3 m}} \sum_{n=0}^{2^{m}-1}\left(n+\frac{1}{2}\right)^{2}\left\|\varphi_{m, n}\right\|_{2}^{2} \\
& +\frac{1}{16} \sum_{j=m}^{\infty} \sum_{k=0}^{2^{j}-1} \frac{1}{2^{3 j}}\left\|\psi_{j, k}\right\|_{2}^{2} \\
& =\frac{1}{2^{3 m}} \sum_{n=0}^{2^{m}-1}\left(n+\frac{1}{2}\right)^{2}+\frac{1}{16} \sum_{j=m}^{\infty} \sum_{k=0}^{2^{j}-1} \frac{1}{2^{3 j}} \\
& =\frac{1}{2^{3 m}} \sum_{n=0}^{2^{m}-1}\left(n^{2}+\frac{1}{4}+n\right)+\frac{1}{16} \sum_{j=m}^{\infty} \frac{1}{2^{3 j}}\left(\sum_{k=0}^{2^{j}-1} 1\right) \\
& =\frac{1}{3}=\|f\|_{2}^{2}
\end{aligned}
$$

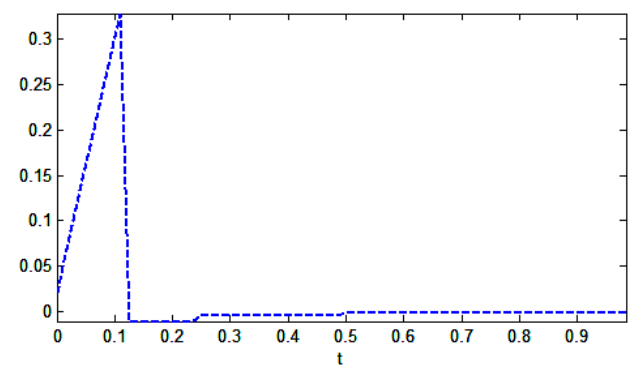

Fig. 10. Graph of decomposed signal $f(t)=t, t \in[0,1)$ with number of discrete points $=64$ at resolution $m=3$ using Haar wavelet matrix.

7.4 Expansion of signal $f(t)=t, t \in[0,3 / 4)$ at resolution $m=0$.

$$
\begin{aligned}
f(t) & =c_{0,0} \varphi_{0,0}(t)+d_{0,0} \psi_{0,0}(t)+d_{1,1} \psi_{1,1}(t)+\sum_{j=1}^{\infty} d_{j, 0} \psi_{j, 0}(t) \\
& +\sum_{j=2}^{\infty} \sum_{k=1}^{3 * 2^{j-2}-1} d_{j, k} \psi_{j, k}(t)
\end{aligned}
$$

$$
\begin{gathered}
\|f\|_{2}^{2}=c_{0,0}^{2}\left\|\varphi_{0,0}\right\|_{2}^{2}+d_{0,0}^{2}\left\|\psi_{0,0}\right\|_{2}^{2}+d_{1,1}^{2}\left\|\psi_{1,1}\right\|_{2}^{2} \\
+\sum_{j=1}^{\infty} d_{j, 0}^{2}\left\|\psi_{j, 0}\right\|_{2}^{2}+\sum_{j=2}^{\infty} \sum_{k=1}^{3 * 2^{j-2}-1} d_{j, k}^{2}\left\|\psi_{j, k}\right\|_{2}^{2} \\
=c_{0,0}^{2}+d_{0,0}^{2}+d_{1,1}^{2}+\sum_{j=1}^{\infty} d_{j, 0}^{2}+\sum_{j=2}^{\infty} \sum_{k=1}^{3 * 2^{j-2}-1} d_{j, k}^{2} \\
\text { L.H.S. }=\|f\|_{2}^{2}=\int_{0}^{3 / 4} t^{2} d t=\frac{9}{2^{6}}
\end{gathered}
$$

$$
\text { R.H.S. }=c_{0,0}^{2}+d_{0,0}^{2}+d_{1,1}^{2}+\sum_{j=1}^{\infty} d_{j, 0}^{2}+\sum_{j=2}^{\infty} \sum_{k=1}^{3 * 2^{j-2}-1} d_{j, k}^{2}
$$

we will calculate each term of this equation seprately with respect to the cases mentioned in table (1).

To evaluate $c_{0,0}^{2}$

$$
c_{0,0}=<f, \varphi_{0,0}>=\int_{0}^{3 / 4} t d t=\frac{9}{32}
$$

Then, $c_{0,0}^{2}=\frac{81}{2^{10}}$

To evaluate $d_{0,0}^{2}$ w.r.t. case-1 of table (1)

$$
\begin{aligned}
d_{0,0} & =\left\langle f, \psi_{0,0}>=\int_{0}^{3 / 4} t * \psi_{0,0}(t) d t\right. \\
& =\int_{0}^{1 / 2} t d t-\int_{1 / 2}^{3 / 4} t d t=-\frac{1}{32}
\end{aligned}
$$

Then, $d_{0,0}^{2}=\frac{1}{2^{10}}$

To evaluate $d_{1,1}^{2}$ w.r.t. case-2 of table (1)

$$
d_{1,1}=<f, \psi_{1,1}>=\int_{1 / 2}^{3 / 4} t * 2^{1 / 2} d t=\frac{5 * 2^{1 / 2}}{32}
$$

Then, $d_{1,1}^{2}=\frac{50}{2^{10}}$

To evaluate $\sum_{j=1}^{\infty} d_{j, 0}^{2}$ w.r.t. case-3 of table (1)

$$
\begin{aligned}
d_{j, 0} & =<f, \psi_{j, 0}>=\int_{0}^{1 / 2^{j}} t * \psi_{j, 0}(t) d t \\
& =\int_{0}^{1 / 2^{j+1}} t * 2^{j / 2} d t-\int_{1 / 2^{j+1}}^{1 / 2^{j}} t * 2^{j / 2} d t \\
& =-\frac{1}{4} \frac{1}{2^{3 j / 2}}
\end{aligned}
$$

Then, $\sum_{j=1}^{\infty} d_{j, 0}^{2}=\sum_{j=1}^{\infty}\left(-\frac{1}{4} \frac{1}{2^{3 j / 2}}\right)^{2}=\frac{1}{16}\left(\sum_{j=1}^{\infty} \frac{1}{2^{3 j}}\right)$

$$
=\frac{1}{16}\left(\frac{1 / 8}{1-1 / 8}\right)=\frac{1}{2^{4} * 7}
$$

To evaluate $\sum_{j=2}^{\infty} \sum_{k=1}^{3 * 2^{j-2}-1} d_{j, k}^{2}$ w.r.t. case-3 of table (1)

$$
\begin{aligned}
d_{j, k} & =<f, \psi_{j, k}>=\int_{k / 2^{j}}^{(k+1) / 2^{j}} t * \psi_{j, k}(t) d t \\
& =\int_{k / 2^{j}}^{(k+1 / 2) / 2^{j}} t * 2^{j / 2} d t-\int_{(k+1 / 2) / 2^{j}}^{(k+1) / 2^{j}} t * 2^{j / 2} d t \\
& =2^{j / 2}\left(\int_{k / 2^{j}}^{(k+1 / 2) / 2^{j}} t d t-\int_{(k+1 / 2) / 2^{j}}^{(k+1) / 2^{j}} t d t\right) \\
& =-\frac{1}{4} \frac{1}{2^{3 j / 2}}
\end{aligned}
$$


Then, $\sum_{j=2}^{\infty} \sum_{k=1}^{3 * 2^{j-2}-1} d_{j, k}^{2}=\sum_{j=2}^{\infty} \sum_{k=1}^{3 * 2^{j-2}-1}\left(-\frac{1}{4} \frac{1}{2^{3 j / 2}}\right)^{2}$

$$
\begin{aligned}
& =\frac{1}{16} \sum_{j=2}^{\infty}\left(\sum_{k=1}^{3 * 2^{j-2}-1} \frac{1}{2^{3 j}}\right) \\
& =\frac{1}{16} \sum_{j=2}^{\infty}\left(\frac{3}{4 * 2^{2 j}}-\frac{1}{2^{3 j}}\right) \\
& =\frac{1}{16}\left\{\frac{3}{4}\left(\sum_{j=2}^{\infty} \frac{1}{2^{2 j}}\right)-\left(\sum_{j=2}^{\infty} \frac{1}{2^{3 j}}\right)\right\} \\
& =\frac{5}{2^{8} * 7}
\end{aligned}
$$

R.H.S $=\frac{81}{2^{10}}+\frac{1}{2^{10}}+\frac{50}{2^{10}}+\frac{1}{2^{4} * 7}+\frac{5}{2^{8} * 7}=\frac{9}{2^{6}}=\|f\|_{2}^{2}$

Hence equation 11 is verified.

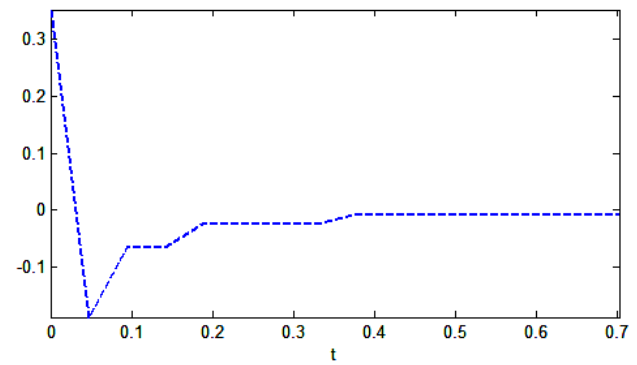

Fig. 11. Graph of decomposed signal $f(t)=t, t \in[0,3 / 4)$ with number of discrete points $=16$ at resolution $m=0$ using Haar wavelet matrix.

\subsection{Expansion of signal $f(t)=\chi_{[0,1]}(t)$, at resolution} $m$.

$$
\begin{aligned}
f(t)=\sum_{n=0}^{2^{m}-1} c_{m, n} \varphi_{m, n}(t)+\sum_{j=m}^{\infty} \sum_{k=0}^{2^{j}-1} d_{j, k} \psi_{j, k}(t) \\
c_{m, n}=<\chi_{[0,1]}, \varphi_{m, n}> \\
=\int_{n / 2^{m}}^{(n+1) / 2^{m}} \chi_{[0,1]}(t) \varphi_{m, n}(t) d t \\
=2^{m / 2} \int_{n / 2^{m}}^{(n+1) / 2^{m}} d t \\
=\frac{1}{2^{m / 2}} \\
d_{j, k}=<\chi_{[0,1]}, \psi_{j, k}> \\
=\int_{k / 2^{j}}^{(k+1) / 2^{j}} \chi_{[0,1]}(t) \psi_{j, k}(t) d t \\
=\int_{k / 2^{j}}^{(k+1) / 2^{j}} \psi_{j, k}(t) d t=0
\end{aligned}
$$

taking square of norm of equation 12 we get

$$
\|f\|_{2}^{2}=\sum_{n=0}^{2^{m}-1} c_{m, n}^{2}+\sum_{j=m}^{\infty} \sum_{k=0}^{2^{j}-1} d_{j, k}^{2}
$$

Now,

$$
\text { R.H.S. }=\sum_{n=0}^{2^{m}-1} c_{m, n}^{2}+\sum_{j=m}^{\infty} \sum_{k=0}^{2^{j}-1} d_{j, k}^{2}
$$

putting values of $c_{m, n}$ and $d_{j, k}$ in this equation, we get

$$
\begin{aligned}
\text { R.H.S. } & =\sum_{n=0}^{2^{m}-1}\left(\frac{1}{2^{m / 2}}\right)^{2}+0 \\
& =1 \\
& =\|f\|_{2}^{2}
\end{aligned}
$$

Hence equation 12 is verified.

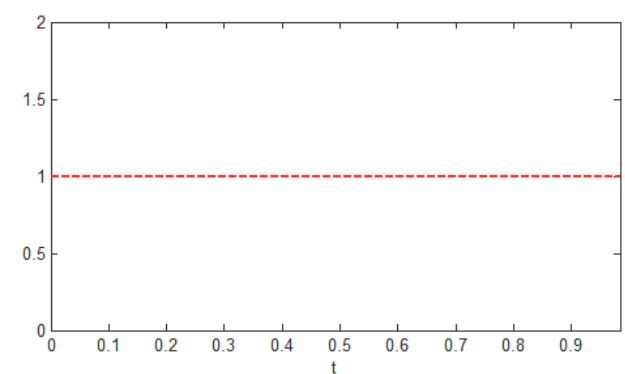

Fig. 12. Graph of signal $f(t)=\chi_{[0,1]}(t)$ with number of discrete points $=64$.

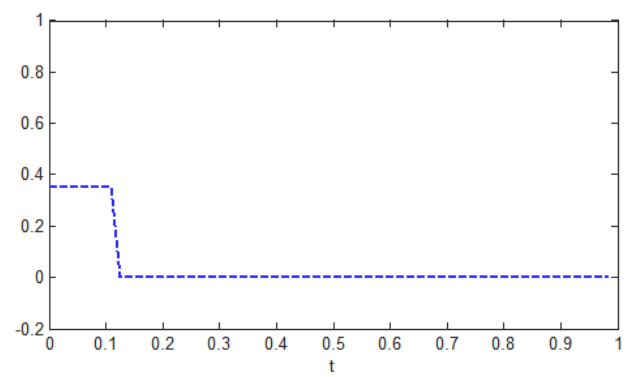

Fig. 13. Graph of decomposed signal $f(t)=\chi_{[0,1]}(t)$ with number of discrete points $=64$ at resolution $m=3$ using Haar wavelet matrix.

7.6 Expansion of signal $f(t)=\chi_{[0,3 / 4]}(t)$, at resolution $m=0$.

$$
\begin{aligned}
f(t) & =c_{0,0} \varphi_{0,0}(t)+d_{0,0} \psi_{0,0}(t)+d_{1,1} \psi_{1,1}(t)+\sum_{j=1}^{\infty} d_{j, 0} \psi_{j, 0}(t) \\
& +\sum_{j=2}^{\infty} \sum_{k=1}^{3 * 2^{j-2}-1} d_{j, k} \psi_{j, k}(t)
\end{aligned}
$$




$$
\begin{gathered}
\|f\|_{2}^{2}=c_{0,0}^{2}+d_{0,0}^{2}+d_{1,1}^{2}+\sum_{j=1}^{\infty} d_{j, 0}^{2}+\sum_{j=2}^{\infty} \sum_{k=1}^{3 * 2^{j-2}-1} d_{j, k}^{2} \\
\text { L.H.S. }=\|f\|_{2}^{2}=\int_{0}^{3 / 4} d t=\frac{3}{4} \\
\text { R.H.S. }=c_{0,0}^{2}+d_{0,0}^{2}+d_{1,1}^{2}+\sum_{j=1}^{\infty} d_{j, 0}^{2}+\sum_{j=2}^{\infty} \sum_{k=1}^{3 * 2^{j-2}-1} d_{j, k}^{2}
\end{gathered}
$$

we will calculate each term of this equation seprately with respect to the cases mentioned in table (1).

To evaluate $c_{0,0}^{2}$

$$
c_{0,0}=<f, \varphi_{0,0}>=\int_{0}^{3 / 4} d t=\frac{3}{4}
$$

Then, $c_{0,0}^{2}=\frac{9}{2^{4}}$

To evaluate $d_{0,0}^{2}$ w.r.t. case-1 of table (1)

$$
\begin{aligned}
d_{0,0} & =<f, \psi_{0,0}>=\int_{0}^{3 / 4} \psi_{0,0}(t) d t \\
& =\int_{0}^{1 / 2} d t-\int_{1 / 2}^{3 / 4} d t=\frac{1}{4}
\end{aligned}
$$

Then, $d_{0,0}^{2}=\frac{1}{2^{4}}$

To evaluate $d_{1,1}^{2}$ w.r.t. case-2 of table (1)

$$
d_{1,1}=<f, \psi_{1,1}>=\int_{1 / 2}^{3 / 4} 2^{1 / 2} d t=\frac{2^{1 / 2}}{4}
$$

Then, $d_{1,1}^{2}=\frac{2}{2^{4}}$

To evaluate $\sum_{j=1}^{\infty} d_{j, 0}^{2}$ w.r.t. case-3 of table (1)

$$
\begin{aligned}
d_{j, 0} & =<f, \psi_{j, 0}>=\int_{0}^{1 / 2^{j}} \psi_{j, 0}(t) d t \\
& =0 \\
\text { Then, } \sum_{j=1}^{\infty} d_{j, 0}^{2} & =0
\end{aligned}
$$

To evaluate $\sum_{j=2}^{\infty} \sum_{k=1}^{3 * 2^{j-2}-1} d_{j, k}^{2}$ w.r.t. case-3 of table ( 1

$$
\begin{aligned}
d_{j, k} & =\left\langle f, \psi_{j, k}>\right. \\
& =\int_{k / 2^{j}}^{(k+1) / 2^{j}} \psi_{j, k}(t) d t=0
\end{aligned}
$$

Then, $\sum_{j=2}^{\infty} \sum_{k=1}^{3 * 2^{j-2}-1} d_{j, k}^{2}=0$

$$
\text { R.H.S }=\frac{9}{2^{4}}+\frac{1}{2^{4}}+\frac{2}{2^{4}}+0+0=\frac{3}{4}=\|f\|_{2}^{2}
$$

Hence equation 13 is verified.

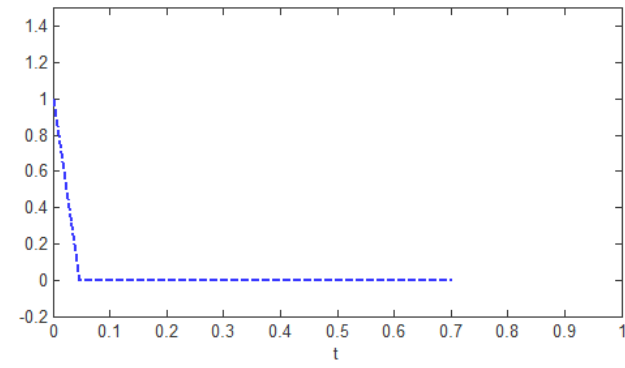

Fig. 14. Graph of decomposed signal $f(t)=t, t \in[0,3 / 4)$ with number of discrete points $=16$ at resolution $m=0$ using Haar wavelet matrix.

\subsection{Expansion of signal}

$$
f\left(t_{1}, t_{2}\right)=t_{1}+t_{2},\left(t_{1}, t_{2}\right) \in[0,1) \times[0,1) \boldsymbol{a t}
$$$$
\text { resolutions } m=m^{\prime}=0 \text {. }
$$

From equation ( 4), double Haar wavelet series for a signal $f \in$ $L^{2}(\mathbb{R} \times \mathbb{R})$ in the region $[0,1) \times[0,1)$ at resolutions $m$ and $m^{\prime}$ is given by,

$$
\begin{array}{r}
f\left(t_{1}, t_{2}\right)=\sum_{n=0}^{2^{m}-1} \sum_{n^{\prime}=0}^{2^{m^{\prime}}-1} c_{m, n ; m^{\prime}, n^{\prime}} \varphi_{m, n ; m^{\prime}, n^{\prime}}\left(t_{1}, t_{2}\right) \\
+\quad \sum_{n=0}^{2^{m}-1} \sum_{j^{\prime}=m^{\prime}}^{\infty} \sum_{k^{\prime}=0}^{2^{j^{\prime}}-1} c_{m, n ; j^{\prime}, k^{\prime}}^{\prime} \varphi_{m, n ; j^{\prime}, k^{\prime}}^{\prime}\left(t_{1}, t_{2}\right) \\
+\quad \sum_{j=m}^{\infty} \sum_{k=0}^{2^{j}-1} \sum_{n^{\prime}=0}^{2^{m^{\prime}}-1} d_{j, k ; m^{\prime}, n^{\prime}}^{\prime} \psi_{j, k ; m^{\prime}, n^{\prime}}^{\prime}\left(t_{1}, t_{2}\right) \\
+\quad \sum_{j=m}^{\infty} \sum_{k=0}^{2^{j}-1} \sum_{j^{\prime}=m^{\prime}}^{\infty} \sum_{k^{\prime}=0}^{2^{j^{\prime}-1}} d_{j, k ; j^{\prime}, k^{\prime}} \psi_{j, k ; j^{\prime}, k^{\prime}}\left(t_{1}, t_{2}\right)
\end{array}
$$

To evaluate $c_{m, n ; m, n^{\prime}}$

$$
\begin{aligned}
c_{m, n ; m, n^{\prime}}= & <f, \varphi_{m, n ; m^{\prime}, n^{\prime}>}> \\
= & \int_{n / 2^{m}}^{(n+1) / 2^{m}} \int_{n^{\prime} / 2^{m^{\prime}}}^{\left(n^{\prime}+1\right) / 2^{m^{\prime}}}\left(t_{1}+t_{2}\right) \\
& * \varphi_{m, n}\left(t_{1}\right) \varphi_{m^{\prime}, n^{\prime}}\left(t_{2}\right) d t_{1} d t_{2} \\
= & \int_{n / 2^{m}}^{(n+1) / 2^{m}} t_{1} \varphi_{m, n}\left(t_{1}\right) d t_{1} \\
& \int_{n^{\prime} / 2^{m^{\prime}}}^{\left(n^{\prime}+1\right) / 2^{m^{\prime}}} \varphi_{m^{\prime}, n^{\prime}}\left(t_{2}\right) d t_{2} \\
& +\int_{n / 2^{m}}^{(n+1) / 2^{m}} \varphi_{m, n}\left(t_{1}\right) d t_{1} \\
& \int_{n^{\prime} / 2^{m^{\prime}}}^{\left(n^{\prime}+1\right) / 2^{m^{\prime}}} t_{2} \varphi_{m^{\prime}, n^{\prime}}\left(t_{2}\right) d t_{2} \\
= & 2^{\left(m+m^{\prime}\right) / 2}\left(\int_{n / 2^{m}}^{(n+1) / 2^{m}} t_{1} d t_{1} \int_{n^{\prime} / 2^{m^{\prime}}}^{\left(n^{\prime}+1\right) / 2^{m^{\prime}}} d t_{2}\right. \\
& \left.+\int_{n / 2^{m}}^{(n+1) / 2^{m}} d t_{1} \int_{n^{\prime} / 2^{m^{\prime}}}^{\left(n^{\prime}+1\right) / 2^{m^{\prime}}} t_{2} d t_{2}\right)
\end{aligned}
$$




$$
=\frac{1}{2} \frac{1}{2^{\left(m+m^{\prime}\right) / 2}}\left\{\frac{(1+2 n)}{2^{m}}+\frac{\left(1+2 n^{\prime}\right)}{2^{m^{\prime}}}\right\}
$$

To evaluate $c_{m, n ; j^{\prime}, k^{\prime}}^{\prime}$

$$
\begin{aligned}
c_{m, n ; j^{\prime}, k^{\prime}}^{\prime}= & <f, \varphi_{m, n ; j^{\prime}, k^{\prime}}^{\prime}> \\
= & \int_{n / 2^{m}}^{(n+1) / 2^{m}} \int_{k^{\prime} / 2^{j^{\prime}}}^{\left(k^{\prime}+1\right) / 2^{j^{\prime}}}\left(t_{1}+t_{2}\right) \\
& * \varphi_{m, n}\left(t_{1}\right) \psi_{j^{\prime}, k^{\prime}}\left(t_{2}\right) d t_{1} d t_{2} \\
= & \int_{n / 2^{m}}^{(n+1) / 2^{m}} t_{1} \varphi_{m, n}\left(t_{1}\right) d t_{1} \\
& \int_{k^{\prime} / 2^{j^{\prime}}}^{\left(k^{\prime}+1\right) / 2^{j^{\prime}}} \psi_{j^{\prime}, k^{\prime}}\left(t_{2}\right) d t_{2} \\
& +\int_{n / 2^{m}}^{(n+1) / 2^{m}} \varphi_{m, n}\left(t_{1}\right) d t_{1} \\
& \int_{k^{\prime} / 2^{j^{\prime}}}^{\left(k^{\prime}+1\right) / 2^{j^{\prime}}} t_{2} \psi_{j^{\prime}, k^{\prime}}\left(t_{2}\right) d t_{2} \\
= & -\frac{1}{4} \frac{1}{2^{m / 2}} \frac{1}{2^{3 j^{\prime} / 2}}
\end{aligned}
$$

To evaluate $d_{j, k ; m^{\prime}, n^{\prime}}^{\prime}$

$$
\begin{aligned}
d_{j, k ; m^{\prime}, n^{\prime}}^{\prime}= & <f, \psi_{j, k ; m^{\prime}, n^{\prime}}^{\prime}> \\
= & \int_{k / 2^{j}}^{(k+1) / 2^{j}} \int_{n^{\prime} / 2^{m^{\prime}}}^{\left(n^{\prime}+1\right) / 2^{m^{\prime}}}\left(t_{1}+t_{2}\right) \\
& * \psi_{j, k}\left(t_{1}\right) \varphi_{m^{\prime}, n^{\prime}}\left(t_{2}\right) d t_{1} d t_{2} \\
= & \int_{k / 2^{j}}^{(k+1) / 2^{j}} t_{1} \psi_{j, k}\left(t_{1}\right) d t_{1} \\
& \int_{n^{\prime} / 2^{m^{\prime}}}^{\left(n^{\prime}+1\right) / 2^{m^{\prime}}} \varphi_{m^{\prime}, n^{\prime}}\left(t_{2}\right) d t_{2} \\
& +\int_{k / 2^{j}}^{(k+1) / 2^{j}} \psi_{j, k}\left(t_{1}\right) d t_{1} \\
& \int_{n^{\prime} / 2^{m^{\prime}}}^{\left(n^{\prime}+1\right) / 2^{m^{\prime}}} t_{2} \varphi_{m^{\prime}, n^{\prime}}\left(t_{2}\right) d t_{2} \\
= & -\frac{1}{4} \frac{1}{2^{m^{\prime} / 2}} \frac{1}{2^{3 j / 2}}
\end{aligned}
$$

To evaluate $d_{j, k ; j^{\prime}, k^{\prime}}$

$$
\begin{aligned}
d_{j, k ; j^{\prime}, k^{\prime}}= & <f, \psi_{j, k ; j^{\prime}, k^{\prime}}> \\
= & \int_{k / 2^{j}}^{(k+1) / 2^{j}} \int_{k^{\prime} / 2^{j^{\prime}}}^{\left(k^{\prime}+1\right) / 2^{j^{\prime}}}\left(t_{1}+t_{2}\right) \\
& * \psi_{j, k}\left(t_{1}\right) \psi_{j^{\prime}, k^{\prime}}\left(t_{2}\right) d t_{1} d t_{2} \\
= & \int_{k / 2^{j}}^{(k+1) / 2^{j}} t_{1} \psi_{j, k}\left(t_{1}\right) d t_{1} \int_{k^{\prime} / 2^{j^{\prime}}}^{\left(k^{\prime}+1\right) / 2^{j^{\prime}}} \psi_{j^{\prime}, k^{\prime}}\left(t_{2}\right) d t_{2} \\
+ & \int_{k / 2^{j}}^{(k+1) / 2^{j}} \psi_{j, k}\left(t_{1}\right) d t_{1} \int_{k^{\prime} / 2^{j^{\prime}}}^{\left(k^{\prime}+1\right) / 2^{j^{\prime}}} t_{2} \psi_{j^{\prime}, k^{\prime}}\left(t_{2}\right) d t_{2} \\
= & 0
\end{aligned}
$$

putting $m=0$ and $m^{\prime}=0$ in equation (14),

$$
\begin{aligned}
f\left(t_{1}, t_{2}\right)= & \sum_{n=0}^{0} \sum_{n^{\prime}=0}^{0} c_{0, n ; 0, n^{\prime}} \varphi_{0, n ; 0, n^{\prime}}\left(t_{1}, t_{2}\right) \\
& +\sum_{n=0}^{0} \sum_{j^{\prime}=0}^{\infty} \sum_{k^{\prime}=0}^{2^{j^{\prime}}-1} c_{0, n ; j^{\prime}, k^{\prime}}^{\prime} \varphi_{0, n ; j^{\prime}, k^{\prime}}^{\prime}\left(t_{1}, t_{2}\right) \\
& +\sum_{j=0}^{\infty} \sum_{k=0}^{2^{j}-1} \sum_{n^{\prime}=0}^{0} d_{j, k ; 0, n^{\prime}}^{\prime} \psi_{j, k ; 0, n^{\prime}}^{\prime}\left(t_{1}, t_{2}\right) \\
& +\sum_{j=0}^{\infty} \sum_{k=0}^{2^{j}-1} \sum_{j^{\prime}=0}^{\infty} \sum_{k^{\prime}=0}^{2^{\prime}-1} d_{j, k ; j^{\prime}, k^{\prime}} \psi_{j, k ; j^{\prime}, k^{\prime}}\left(t_{1}, t_{2}\right) \\
= & c_{0,0 ; 0,0} \varphi_{0,0 ; 0,0}\left(t_{1}, t_{2}\right) \\
& +\sum_{j^{\prime}=0}^{\infty} \sum_{k^{\prime}=0}^{2^{j^{\prime}}-1} c_{0,0 ; j^{\prime}, k^{\prime}}^{\prime} \varphi_{0,0 ; j^{\prime}, k^{\prime}}^{\prime}\left(t_{1}, t_{2}\right) \\
& +\sum_{j=0}^{\infty} \sum_{k=0}^{2^{j}-1} d_{j, k ; 0,0}^{\prime} \psi_{j, k ; 0,0}^{\prime}\left(t_{1}, t_{2}\right) \\
& +\sum_{j=0}^{\infty} \sum_{k=0}^{2^{j}-1} \sum_{j^{\prime}=0}^{\infty} \sum_{k^{\prime}=0}^{2^{\prime}-1} d_{j, k ; j^{\prime}, k^{\prime}} \psi_{j, k ; j^{\prime}, k^{\prime}}\left(t_{1}, t_{2}\right)
\end{aligned}
$$

By equation (15),

$$
c_{0,0 ; 0,0}=1
$$

By equation (31),

$$
c_{0,0 ; j^{\prime}, k^{\prime}}^{\prime}=-\frac{1}{4} \frac{1}{2^{3 j^{\prime} / 2}}
$$

By equation (31),

$$
d_{j, k ; 0,0}^{\prime}=-\frac{1}{4} \frac{1}{2^{3 j / 2}}
$$

$$
\begin{aligned}
f\left(t_{1}, t_{2}\right) & =\varphi_{0,0 ; 0,0}\left(t_{1}, t_{2}\right) \\
& -\frac{1}{4} \sum_{j^{\prime}=0}^{\infty} \sum_{k^{\prime}=0}^{2^{j^{\prime}}-1} \frac{1}{2^{3 j^{\prime} / 2}} \varphi_{0,0 ; j^{\prime}, k^{\prime}}^{\prime}\left(t_{1}, t_{2}\right) \\
& -\frac{1}{4} \sum_{j=0}^{\infty} \sum_{k=0}^{2^{j}-1} \frac{1}{2^{3 j / 2}} \psi_{j, k ; 0,0}^{\prime}\left(t_{1}, t_{2}\right)
\end{aligned}
$$




$$
\begin{aligned}
\|f\|_{2}^{2} & =\left\|\varphi_{0,0 ; 0,0}\right\|_{2}^{2} \\
& +\left(-\frac{1}{4}\right)^{2} \sum_{j^{\prime}=0}^{\infty} \sum_{k^{\prime}=0}^{2^{j^{\prime}}-1}\left(\frac{1}{2^{3 j^{\prime} / 2}}\right)^{2}\left\|\varphi_{0,0 ; j^{\prime}, k^{\prime}}^{\prime}\right\|_{2}^{2} \\
& +\left(-\frac{1}{4}\right)^{2} \sum_{j=0}^{\infty} \sum_{k=0}^{2^{j}-1}\left(\frac{1}{2^{3 j / 2}}\right)^{2}\left\|\psi_{j, k ; 0,0}^{\prime}\right\|_{2}^{2} \\
& =\left\|\varphi_{0,0 ; 0,0}\right\|_{2}^{2} \\
& +\frac{1}{2^{4}} \sum_{j^{\prime}=0}^{\infty} \sum_{k^{\prime}=0}^{2^{\prime}-1} \frac{1}{2^{3 j^{\prime}}}\left\|\varphi_{0,0 ; j^{\prime}, k^{\prime}}^{\prime}\right\|_{2}^{2} \\
& +\frac{1}{2^{4}} \sum_{j=0}^{\infty} \sum_{k=0}^{2^{j}-1} \frac{1}{2^{3 j}}\left\|\psi_{j, k ; 0,0}^{\prime}\right\|_{2}^{2}
\end{aligned}
$$

To evaluate $\left\|\varphi_{m, n ; m^{\prime}, n^{\prime}}\right\|_{2}^{2}$

$$
\begin{aligned}
\left\|\varphi_{m, n ; m^{\prime}, n^{\prime}}\right\|_{2}^{2}= & \int_{-\infty}^{\infty} \int_{-\infty}^{\infty}\left|\varphi_{m, n ; m^{\prime}, n^{\prime}}\left(t_{1}, t_{2}\right)\right|^{2} d t_{1} d t_{2} \\
= & \int_{n / 2^{m}}^{(n+1) / 2^{m}}\left|\varphi_{m, n}\left(t_{1}\right)\right|^{2} d t_{1} \\
& \int_{n^{\prime} / 2^{m^{\prime}}}^{\left(n^{\prime}+1\right) / 2^{m^{\prime}}}\left|\varphi_{m^{\prime}, n^{\prime}}\left(t_{2}\right)\right|^{2} d t_{2} \\
= & \int_{n / 2^{m}}^{(n+1) / 2^{m}}\left(2^{m / 2}\right)^{2} d t_{1} \\
& \int_{n^{\prime} / 2^{m^{\prime}}}^{\left(n^{\prime}+1\right) / 2^{m^{\prime}}}\left(2^{m^{\prime} / 2}\right)^{2} d t_{2}=1
\end{aligned}
$$

To evaluate $\left\|\varphi_{m, n ; j^{\prime}, k^{\prime}}^{\prime}\right\|_{2}^{2}$

$$
\begin{aligned}
\left\|\varphi_{m, n ; j^{\prime}, k^{\prime}}^{\prime}\right\|_{2}^{2}= & \int_{-\infty}^{\infty} \int_{-\infty}^{\infty}\left|\varphi_{m, n ; j^{\prime}, k^{\prime}}^{\prime}\left(t_{1}, t_{2}\right)\right|^{2} d t_{1} d t_{2} \\
= & \int_{n / 2^{m}}^{(n+1) / 2^{m}}\left|\varphi_{m, n}\left(t_{1}\right)\right|^{2} d t_{1} \\
& \int_{k^{\prime} / 2^{j^{\prime}}}^{\left(k^{\prime}+1\right) / 2^{j^{\prime}}}\left|\psi_{j^{\prime}, k^{\prime}}\left(t_{2}\right)\right|^{2} d t_{2} \\
= & \int_{n / 2^{m}}^{(n+1) / 2^{m}}\left(2^{m / 2}\right)^{2} d t_{1}\left(\int_{k^{\prime} / 2^{j^{\prime}}}^{\left(k^{\prime}+1 / 2\right) / 2^{j^{\prime}}}\right. \\
& \left.\left(2^{j^{\prime} / 2}\right)^{2} d t_{2}+\int_{\left(k^{\prime}+1 / 2\right) / 2^{j^{\prime}}}^{\left(k^{\prime}+1\right) / 2^{j^{\prime}}}\left(-2^{j^{\prime} / 2}\right)^{2} d t_{2}\right) \\
= & 1
\end{aligned}
$$

To evaluate $\left\|\psi_{j, k ; m^{\prime}, n^{\prime}}^{\prime}\right\|_{2}^{2}$

$$
\begin{aligned}
\left\|\psi_{j, k ; m^{\prime}, n^{\prime}}^{\prime}\right\|_{2}^{2}= & \int_{-\infty}^{\infty} \int_{-\infty}^{\infty}\left|\psi_{j, k ; m^{\prime}, n^{\prime}}^{\prime}\left(t_{1}, t_{2}\right)\right|^{2} d t_{1} d t_{2} \\
= & \int_{k / 2^{j}}^{(k+1) / 2^{j}}\left|\psi_{j, k}\left(t_{1}\right)\right|^{2} d t_{1} \\
& \int_{n^{\prime} / 2^{m^{\prime}}}^{\left(n^{\prime}+1\right) / 2^{m^{\prime}}}\left|\varphi_{m^{\prime}, n^{\prime}}\left(t_{2}\right)\right|^{2} d t_{2} \\
= & \left(\int_{k / 2^{j}}^{(k+1 / 2) / 2^{j}}\left(2^{j / 2}\right)^{2} d t_{1}\right. \\
& \left.+\int_{(k+1 / 2) / 2^{j}}^{(k+1) / 2^{j}}\left(-2^{j / 2}\right)^{2} d t_{1}\right) \\
& \int_{n^{\prime} / 2^{m^{\prime}}}^{\left(n^{\prime}+1\right) / 2^{m^{\prime}}}\left(2^{m^{\prime} / 2}\right)^{2} d t_{2} \\
= & 1
\end{aligned}
$$

To evaluate $\left\|\psi_{j, k ; j^{\prime}, k^{\prime}}\right\|_{2}^{2}$

$$
\begin{aligned}
\left\|\psi_{j, k ; j^{\prime}, k^{\prime}}\right\|_{2}^{2}= & \int_{-\infty}^{\infty} \int_{-\infty}^{\infty}\left|\psi_{j, k ; j^{\prime}, k^{\prime}}\left(t_{1}, t_{2}\right)\right|^{2} d t_{1} d t_{2} \\
= & \int_{k / 2 j}^{(k+1) / 2^{j}}\left|\psi_{j, k}\left(t_{1}\right)\right|^{2} d t_{1} \\
& \int_{k^{\prime} / 2^{j^{\prime}}}^{\left(k^{\prime}+1\right) / 2^{j^{\prime}}}\left|\psi_{j^{\prime}, k^{\prime}}\left(t_{2}\right)\right|^{2} d t_{2} \\
= & \left(\int_{k / 2^{j}}^{(k+1 / 2) / 2^{j}}\left(2^{j / 2}\right)^{2} d t_{1}\right. \\
& \left.+\int_{(k+1 / 2) / 2^{j}}^{(k+1) / 2^{j}}\left(-2^{j / 2}\right)^{2} d t_{1}\right) \\
& *\left(\int_{k^{\prime} / 2^{j^{\prime}}}^{\left(k^{\prime}+1 / 2\right) / 2^{j^{\prime}}}\left(2^{j^{\prime} / 2}\right)^{2} d t_{2}\right. \\
& \left.+\int_{\left(k^{\prime}+1 / 2\right) / 2^{j^{\prime}}}^{\left(k^{\prime}+1\right) / 2^{j^{\prime}}}\left(-2^{j^{\prime} / 2}\right)^{2} d t_{2}\right) \\
= & 1
\end{aligned}
$$

By equation ( 211, ( 22) and ( 23), it is clear that, $\left\|\varphi_{0,0 ; 0,0}\right\|_{2}^{2}=1,\left\|\varphi_{0,0 ; j^{\prime}, k^{\prime}}^{\prime}\right\|_{2}^{2}=1$,

$\left\|\psi_{j, k ; 0,0}^{\prime}\right\|_{2}^{2}=1$

taking L.H.S. of equation (20),

$$
\begin{aligned}
\text { L.H.S. } & =\|f\|_{2}^{2}=\int_{0}^{1} \int_{0}^{1}\left(t_{1}+t_{2}\right)^{2} d t_{1} d t_{2} \\
& =\frac{7}{6}
\end{aligned}
$$


taking R.H.S. of equation (20),

$$
\begin{aligned}
\text { R.H.S }= & \left\|\varphi_{0,0 ; 0,0}\right\|_{2}^{2} \\
& +\frac{1}{2^{4}} \sum_{j^{\prime}=0}^{\infty} \sum_{k^{\prime}=0}^{2^{j^{\prime}}-1} \frac{1}{2^{3 j^{\prime}}}\left\|\varphi_{0,0 ; j^{\prime}, k^{\prime}}^{\prime}\right\|_{2}^{2} \\
& +\frac{1}{2^{4}} \sum_{j=0}^{\infty} \sum_{k=0}^{2^{j}-1} \frac{1}{2^{3 j}}\left\|\psi_{j, k ; 0,0}^{\prime}\right\|_{2}^{2}
\end{aligned}
$$

putting values of $\left\|\varphi_{0,0 ; 0,0}\right\|_{2}^{2},\left\|\varphi_{0,0 ; j^{\prime}, k^{\prime}}^{\prime}\right\|_{2}^{2}$,

$\left\|\psi_{j, k ; 0,0}^{\prime}\right\|_{2}^{2}$, we get,

$$
\begin{aligned}
R . H . S & =1+\frac{1}{2^{4}} \sum_{j^{\prime}=0}^{\infty} \sum_{k^{\prime}=0}^{2^{j^{\prime}}-1} \frac{1}{2^{3 j^{\prime}}}+\frac{1}{2^{4}} \sum_{j=0}^{\infty} \sum_{k=0}^{2^{j}-1} \frac{1}{2^{3 j}} \\
& =1+\frac{1}{2^{4}} \sum_{j^{\prime}=0}^{\infty} \frac{1}{2^{3 j^{\prime}}}\left(\sum_{k^{\prime}=0}^{2^{j^{\prime}}-1} 1\right)+\frac{1}{2^{4}} \sum_{j=0}^{\infty} \frac{1}{2^{3 j}}\left(\sum_{k=0}^{2^{j}-1} 1\right) \\
& =1+\frac{1}{2^{4}}\left(\sum_{j^{\prime}=0}^{\infty} \frac{1}{2^{2 j^{\prime}}}\right)+\frac{1}{2^{4}}\left(\sum_{j=0}^{\infty} \frac{1}{2^{2 j}}\right) \\
& =1+\frac{1}{2^{4}}\left(\frac{1}{1-1 / 2^{2}}\right)+\frac{1}{2^{4}}\left(\frac{1}{1-1 / 2^{2}}\right) \\
& =\frac{7}{6}
\end{aligned}
$$

By equation (31) and (32), we get

$$
\text { L.H.S = R.H.S }
$$

Hence, double wavelet series is verified.

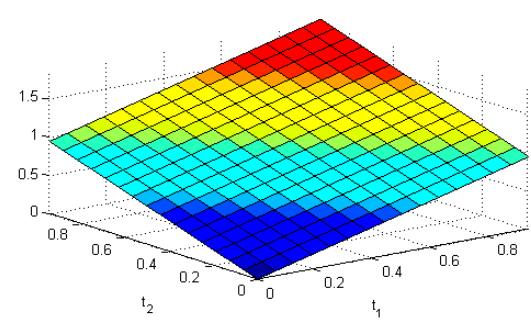

Fig. 15. Graph of signal $f\left(t_{1}, t_{2}\right)=t_{1}+t_{2}, t \in[0,1) \times[0,1)$ with order of matrix $=16$

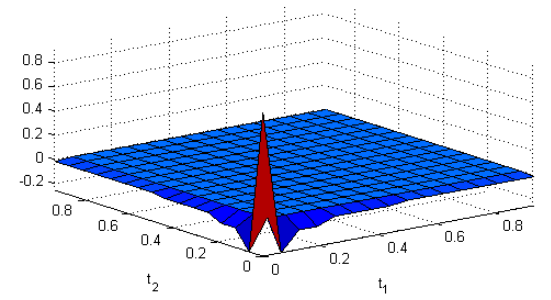

Fig. 16. Graph of decomposed signal $f\left(t_{1}, t_{2}\right)=t_{1}+t_{2}, t \in[0,1) \times$ $[0,1)$ with order of matrix $=16$ at resolutions $m_{1}=m_{2}=0$

\subsection{Expansion of signal}

$f\left(t_{1}, t_{2}\right)=t_{1}+t_{2},\left(t_{1}, t_{2}\right) \in[0,3 / 4) \times[0,3 / 4) \boldsymbol{a t}$ resolutions $m=m^{\prime}=0$.

From equation ( 5 , double Haar wavelet series for a signal $f \in$ $L^{2}(\mathbb{R} \times \mathbb{R})$ in the region $[0,3 / 4) \times[0.3 / 4)$ at resolutions $m=$ $m^{\prime}=0$ is given by,

$$
\begin{aligned}
& f\left(t_{1}, t_{2}\right)=c_{0,0 ; 0,0} \varphi_{0,0 ; 0,0}\left(t_{1}, t_{2}\right)+c_{0,0 ; 0,0}^{\prime} \varphi_{0,0 ; 0,0}^{\prime}\left(t_{1}, t_{2}\right) \\
& +c_{0,0 ; 1,1}^{\prime} \varphi_{0,0 ; 1,1}^{\prime}\left(t_{1}, t_{2}\right)+\sum_{j^{\prime}=1}^{\infty} c_{0,0 ; j^{\prime}, 0}^{\prime} \varphi_{0,0 ; j^{\prime}, 0}^{\prime}\left(t_{1}, t_{2}\right) \\
& +\sum_{j^{\prime}=2}^{\infty} \sum_{k^{\prime}=1}^{3 * j^{j^{\prime}-2}-1} c_{0,0 ; j^{\prime}, k^{\prime}}^{\prime} \varphi_{0,0 ; j^{\prime}, k^{\prime}}^{\prime}\left(t_{1}, t_{2}\right) \\
& +d_{0,0 ; 0,0}^{\prime} \psi_{0,0 ; 0,0}^{\prime}\left(t_{1}, t_{2}\right)+d_{0,0 ; 0,0} \psi_{0,0 ; 0,0}\left(t_{1}, t_{2}\right) \\
& +d_{0,0 ; 1,1} \psi_{0,0 ; 1,1}\left(t_{1}, t_{2}\right)+\sum_{j^{\prime}=1}^{\infty} d_{0,0 ; j^{\prime}, 0} \psi_{0,0 ; j^{\prime}, 0}\left(t_{1}, t_{2}\right) \\
& +\sum_{j^{\prime}=2}^{\infty} \sum_{k^{\prime}=1}^{3 * 2^{j^{\prime}-2}-1} d_{0,0 ; j^{\prime}, k^{\prime}} \psi_{0,0 ; j^{\prime}, k^{\prime}}\left(t_{1}, t_{2}\right) \\
& +d_{1,1 ; 0,0}^{\prime} \psi_{1,1 ; 0,0}^{\prime}\left(t_{1}, t_{2}\right)+d_{1,1 ; 0,0} \psi_{1,1 ; 0,0}\left(t_{1}, t_{2}\right) \\
& +d_{1,1 ; 1,1} \psi_{1,1 ; 1,1}\left(t_{1}, t_{2}\right)+\sum_{j^{\prime}=1}^{\infty} d_{1,1 ; j^{\prime}, 0} \psi_{1,1 ; j^{\prime}, 0}\left(t_{1}, t_{2}\right)
\end{aligned}
$$

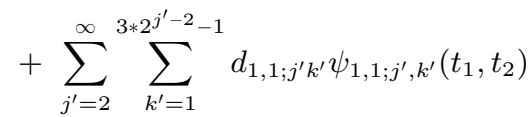

$$
\begin{aligned}
& +\sum_{j=1}^{\infty} d_{j, 0 ; 0,0}^{\prime} \psi_{j, 0 ; 0,0}^{\prime}\left(t_{1}, t_{2}\right) \\
& +\sum_{j=1}^{\infty} d_{j, 0 ; 0,0} \psi_{j, 0 ; 0,0}\left(t_{1}, t_{2}\right)+\sum_{j=1}^{\infty} d_{j, 0 ; 1,1} \psi_{j, 0 ; 1,1}\left(t_{1}, t_{2}\right) \\
& +\sum_{j=1}^{\infty} \sum_{j^{\prime}=1}^{\infty} d_{j, 0 ; j^{\prime}, 0} \psi_{j, 0 ; j^{\prime}, 0}\left(t_{1}, t_{2}\right) \\
& +\sum_{j=1}^{\infty} \sum_{j^{\prime}=2}^{\infty} \sum_{k^{\prime}=1}^{3 * 2^{j^{\prime}-2}-1} d_{j, 0 ; j^{\prime}, k^{\prime}} \psi_{j, 0 ; j^{\prime}, k^{\prime}}\left(t_{1}, t_{2}\right) \\
& +\sum_{j=2}^{\infty} \sum_{k=1}^{3 * 2^{j-2}-1} d_{j, k ; 0,0}^{\prime} \psi_{j, k ; 0,0}^{\prime}\left(t_{1}, t_{2}\right) \\
& +\sum_{j=2}^{\infty} \sum_{k=1}^{3 * 2^{j-2}-1} d_{j, k ; 0,0} \psi_{j, k ; 0,0}\left(t_{1}, t_{2}\right) \\
& +\sum_{j=2}^{\infty} \sum_{k=1}^{3 * 2^{j-2}-1} d_{j, k ; 1,1} \psi_{j, k ; 1,1}\left(t_{1}, t_{2}\right) \\
& +\sum_{j=2}^{\infty} \sum_{k=1}^{3 * 2^{j-2}} \sum_{j^{\prime}=1}^{\infty} d_{j, k ; j^{\prime}, 0} \psi_{j, k ; j^{\prime}, 0}\left(t_{1}, t_{2}\right) \\
& +\sum_{j=2}^{\infty} \sum_{k=1}^{3 * 2^{j-2}-1} \sum_{j^{\prime}=2}^{\infty} \sum_{k^{\prime}=1}^{3 * j^{j^{\prime}-2}-1} d_{j, k ; j^{\prime}, k^{\prime}} \psi_{j, k ; j^{\prime}, k^{\prime}}\left(t_{1}, t_{2}\right)
\end{aligned}
$$


taking square of norm of both sides,

$$
\begin{aligned}
& \|f\|_{2}^{2}=c_{0,0 ; 0,0}^{2}\left\|\varphi_{0,0 ; 0,0}\right\|_{2}^{2} \\
& +c^{\prime 2}{ }_{0,0 ; 0,0}\left\|\varphi_{0,0 ; 0,0}^{\prime}\right\|_{2}^{2} \\
& +c_{0,0 ; 1,1}^{\prime 2}\left\|\varphi_{0,0 ; 1,1}^{\prime}\right\|_{2}^{2} \\
& +\sum_{j^{\prime}=1}^{\infty} c_{0,0 ; j^{\prime}, 0}^{\prime 2}\left\|\varphi_{0,0 ; j^{\prime}, 0}^{\prime}\right\|_{2}^{2} \\
& +\sum_{j^{\prime}=2}^{\infty} \sum_{k^{\prime}=1}^{3 * 2^{j^{\prime}-2}-1} c_{0,0 ; j^{\prime}, k^{\prime}}^{\prime 2}\left\|\varphi_{0,0 ; j^{\prime}, k^{\prime}}^{\prime}\right\|_{2}^{2} \\
& +d_{0,0 ; 0,0}^{\prime 2}\left\|\psi_{0,0 ; 0,0}^{\prime}\right\|_{2}^{2} \\
& +d_{0,0 ; 0,0}^{2}\left\|\psi_{0,0 ; 0,0}\right\|_{2}^{2} \\
& +d_{0,0 ; 1,1}^{2}\left\|\psi_{0,0 ; 1,1}\right\|_{2}^{2} \\
& +\sum_{j^{\prime}=1}^{\infty} d_{0,0 ; j^{\prime}, 0}^{2}\left\|\psi_{0,0 ; j^{\prime}, 0}\right\|_{2}^{2} \\
& +\sum_{j^{\prime}=2}^{\infty} \sum_{k^{\prime}=1}^{3 * 2^{j^{\prime}-2}-1} d_{0,0 ; j^{\prime}, k^{\prime}}^{2}\left\|\psi_{0,0 ; j^{\prime}, k^{\prime}}\right\|_{2}^{2} \\
& +d_{1,1 ; 0,0}^{\prime 2}\left\|\psi_{1,1 ; 0,0}^{\prime}\right\|_{2}^{2} \\
& +d_{1,1 ; 0,0}^{2}\left\|\psi_{1,1 ; 0,0}\right\|_{2}^{2} \\
& +d_{1,1 ; 1,1}^{2}\left\|\psi_{1,1 ; 1,1}\right\|_{2}^{2} \\
& +\sum_{j^{\prime}=1}^{\infty} d_{1,1 ; j^{\prime}, 0}^{2}\left\|\psi_{1,1 ; j^{\prime}, 0}\right\|_{2}^{2} \\
& +\sum_{j^{\prime}=2}^{\infty} \sum_{k^{\prime}=1}^{3 * 2^{j^{\prime}-2}-1} d_{1,1 ; j^{\prime} k^{\prime}}^{2}\left\|\psi_{1,1 ; j^{\prime}, k^{\prime}}\right\|_{2}^{2} \\
& +\sum_{j=1}^{\infty}{d^{\prime}}_{j, 0 ; 0,0}^{2}\left\|\psi_{j, 0 ; 0,0}^{\prime}\right\|_{2}^{2} \\
& +\sum_{j=1}^{\infty} d_{j, 0 ; 0,0}^{2}\left\|\psi_{j, 0 ; 0,0}\right\|_{2}^{2} \\
& +\sum_{j=1}^{\infty} d_{j, 0 ; 1,1}^{2}\left\|\psi_{j, 0 ; 1,1}\right\|_{2}^{2} \\
& +\sum_{j=1}^{\infty} \sum_{j^{\prime}=1}^{\infty} d_{j, 0 ; j^{\prime}, 0}^{2}\left\|\psi_{j, 0 ; j^{\prime}, 0}\right\|_{2}^{2} \\
& +\sum_{j=1}^{\infty} \sum_{j^{\prime}=2}^{\infty} \sum_{k^{\prime}=1}^{3 * 2^{j^{\prime}-2}-1} d_{j, 0 ; j^{\prime}, k^{\prime}}^{2}\left\|\psi_{j, 0 ; j^{\prime}, k^{\prime}}\right\|_{2}^{2} \\
& +\sum_{j=2}^{\infty} \sum_{k=1}^{3 * 2^{j-2}-1} d_{j, k ; 0,0}^{\prime 2}\left\|\psi_{j, k ; 0,0}^{\prime}\right\|_{2}^{2} \\
& +\sum_{j=2}^{\infty} \sum_{k=1}^{3 * 2^{j-2}-1} d_{j, k ; 0,0}^{2}\left\|\psi_{j, k ; 0,0}\right\|_{2}^{2} \\
& +\sum_{j=2}^{\infty} \sum_{k=1}^{3 * 2^{j-2}-1} d_{j, k ; 1,1}^{2}\left\|\psi_{j, k ; 1,1}\right\|_{2}^{2} \\
& +\sum_{j=2}^{\infty} \sum_{k=1}^{3 * 2^{j-2}} \sum_{j^{\prime}=1}^{\infty} d_{j, k ; j^{\prime}, 0}^{2}\left\|\psi_{j, k ; j^{\prime}, 0}\right\|_{2}^{2} \\
& +\sum_{j=2}^{\infty} \sum_{k=1}^{3 * 2^{j-2}} \sum_{j^{\prime}=2}^{\infty} \sum_{k^{\prime}=1}^{3 * 2^{j^{\prime}-2}-1} d_{j, k ; j^{\prime}, k^{\prime}}^{2}\left\|\psi_{j, k ; j^{\prime}, k^{\prime}}\right\|_{2}^{2}
\end{aligned}
$$

$$
\begin{aligned}
& =c_{0,0 ; 0,0}^{2}+c^{\prime 2}{ }_{0,0 ; 0,0}+c_{0,0 ; 1,1}^{\prime 2}+\sum_{j^{\prime}=1}^{\infty} c_{0,0 ; j^{\prime}, 0}^{\prime 2} \\
& +\sum_{j^{\prime}=2}^{\infty} \sum_{k^{\prime}=1}^{3 * 2^{j^{\prime}-2}-1} c_{0,0 ; j^{\prime}, k^{\prime}}^{\prime 2}+d_{0,0 ; 0,0}^{2}+d_{0,0 ; 0,0}^{2} \\
& +d_{0,0 ; 1,1}^{2}+\sum_{j^{\prime}=1}^{\infty} d_{0,0 ; j^{\prime}, 0}^{2}+\sum_{j^{\prime}=2}^{\infty} \sum_{k^{\prime}=1}^{3 * 2^{j^{\prime}}-2} d_{0,0 ; j^{\prime}, k^{\prime}}^{2} \\
& +d_{1,1 ; 0,0}^{\prime 2}+d_{1,1 ; 0,0}^{2}+d_{1,1 ; 1,1}^{2}+\sum_{j^{\prime}=1}^{\infty} d_{1,1 ; j^{\prime}, 0}^{2} \\
& +\sum_{j^{\prime}=2}^{\infty} \sum_{k^{\prime}=1}^{3 * 2^{j^{\prime}-2}-1} d_{1,1 ; j^{\prime}, k^{\prime}}^{2}+\sum_{j=1}^{\infty}{d^{\prime 2}}_{j, 0 ; 0,0}^{2}+\sum_{j=1}^{\infty} d_{j, 0 ; 0,0}^{2} \\
& +\sum_{j=1}^{\infty} d_{j, 0 ; 1,1}^{2}+\sum_{j=1}^{\infty} \sum_{j^{\prime}=1}^{\infty} d_{j, 0 ; j^{\prime}, 0}^{2}+\sum_{j=1}^{\infty} \sum_{j^{\prime}=2}^{\infty} \sum_{k^{\prime}=1}^{3 * 2^{j^{\prime}-2}-1} d_{j, 0 ; j^{\prime}, k^{\prime}}^{2} \\
& +\sum_{j=2}^{\infty} \sum_{k=1}^{3 * 2^{j-2}-1} d^{\prime 2}{ }_{j, k ; 0,0}+\sum_{j=2}^{\infty} \sum_{k=1}^{3 * 2^{j-2}-1} d_{j, k ; 0,0}^{2} \\
& +\sum_{j=2}^{\infty} \sum_{k=1}^{3 * 2^{j-2}-1} d_{j, k ; 1,1}^{2}+\sum_{j=2}^{\infty} \sum_{k=1}^{3 * 2^{j-2}-1} \sum_{j^{\prime}=1}^{\infty} d_{j, k ; j^{\prime}, 0}^{2} \\
& +\sum_{j=2}^{\infty} \sum_{k=1}^{3 * 2^{j-2}} \sum_{j^{\prime}=2}^{\infty} \sum_{k^{\prime}=1}^{3 * 2^{j^{\prime}-2}-1} d_{j, k ; j^{\prime}, k^{\prime}}^{2}
\end{aligned}
$$

$\because\left\|\varphi_{m, n ; m^{\prime}, n^{\prime}}\right\|_{2}^{2}=1,\left\|\varphi_{m, n ; j^{\prime}, k^{\prime}}^{\prime}\right\|_{2}^{2}=1,\left\|\psi_{j, k ; j^{\prime}, k^{\prime}}\right\|_{2}^{2}=1$, $\left\|\psi_{j, k ; m^{\prime}, n^{\prime}}^{\prime}\right\|_{2}^{2}=1$

taking L.H.S. of the equation,

$$
\begin{aligned}
& \text { L.H.S. }=\|f\|_{2}^{2}=\int_{0}^{3 / 4} \int_{0}^{3 / 4}\left(t_{1}+t_{2}\right)^{2} d t_{1} d t_{2} \\
& =\frac{189}{2^{9}}
\end{aligned}
$$

$$
\begin{aligned}
\text { R.H.S. } & =c_{0,0 ; 0,0}^{2}+c_{0,0 ; 0,0}^{\prime 2}+c_{0,0 ; 1,1}^{\prime 2}+\sum_{j^{\prime}=1}^{\infty}{c^{\prime}}_{0,0 ; j^{\prime}, 0}^{2} \\
& +\sum_{j^{\prime}=2}^{\infty} \sum_{k^{\prime}=1}^{3 * 2 j^{j^{\prime}-2}-1} c_{0,0 ; j^{\prime}, k^{\prime}}^{\prime 2}+d_{0,0 ; 0,0}^{\prime 2}+d_{0,0 ; 0,0}^{2} \\
& +d_{0,0 ; 1,1}^{2}+\sum_{j^{\prime}=1}^{\infty} d_{0,0 ; j^{\prime}, 0}^{2}+\sum_{j^{\prime}=2}^{\infty} \sum_{k^{\prime}=1}^{3 * 2^{j^{\prime}-2}-1} d_{0,0 ; j^{\prime}, k^{\prime}}^{2} \\
& +d_{1,1 ; 0,0}^{\prime 2}+d_{1,1 ; 0,0}^{2}+d_{1,1 ; 1,1}^{2}+\sum_{j^{\prime}=1}^{\infty} d_{1,1 ; j^{\prime}, 0}^{2} \\
& +\sum_{j^{\prime}=2}^{\infty} \sum_{k^{\prime}=1}^{3 * 2^{j^{\prime}-2}-1} d_{1,1 ; j^{\prime}, k^{\prime}}^{2}+\sum_{j=1}^{\infty} d^{\prime 2}{ }_{j, 0 ; 0,0}+\sum_{j=1}^{\infty} d_{j, 0 ; 0,0}^{2} \\
& +\sum_{j=1}^{\infty} d_{j, 0 ; 1,1}^{2}+\sum_{j=1}^{\infty} \sum_{j^{\prime}=1}^{\infty} d_{j, 0 ; j^{\prime}, 0}^{2}
\end{aligned}
$$




$$
\begin{aligned}
& +\sum_{j=1}^{\infty} \sum_{j^{\prime}=2}^{\infty} \sum_{k^{\prime}=1}^{3 * 2^{j^{\prime}-2}-1} d_{j, 0 ; j^{\prime}, k^{\prime}}^{2}+\sum_{j=2}^{\infty} \sum_{k=1}^{3 * 2^{j-2}-1} d_{j, k ; 0,0}^{2} \\
& +\sum_{j=2}^{\infty} \sum_{k=1}^{3 * 2^{j-2}-1} d_{j, k ; 0,0}^{2}+\sum_{j=2}^{\infty} \sum_{k=1}^{3 * 2^{j-2}-1} d_{j, k ; 1,1}^{2} \\
& +\sum_{j=2}^{\infty} \sum_{k=1}^{3 * 2^{j-2}-1} \sum_{j^{\prime}=1}^{\infty} d_{j, k ; j^{\prime}, 0}^{2} \\
& +\sum_{j=2}^{\infty} \sum_{k=1}^{3 * 2^{j-2}-1} \sum_{j^{\prime}=2}^{\infty} \sum_{k^{\prime}=1}^{3 * 2^{j^{\prime}-2}-1} d_{j, k ; j^{\prime}, k^{\prime}}^{2}
\end{aligned}
$$

we will calculate each term of this equation seprately with respect to the cases mentioned in table ([1).

To evaluate $c_{0,0 ; 0,0}^{2}$

$$
\begin{aligned}
c_{0,0 ; 0,0}= & <f, \varphi_{0,0 ; 0,0}> \\
= & \int_{0}^{3 / 4} \int_{0}^{3 / 4}\left(t_{1}+t_{2}\right) \varphi_{0,0}\left(t_{1}\right) \varphi_{0,0}\left(t_{2}\right) d t_{1} d t_{2} \\
= & \int_{0}^{3 / 4} t_{1} \varphi_{0,0}\left(t_{1}\right) d t_{1} \int_{0}^{3 / 4} \varphi_{0,0}\left(t_{2}\right) d t_{2} \\
& +\int_{0}^{3 / 4} \varphi_{0,0}\left(t_{1}\right) d t_{1} \int_{0}^{3 / 4} t_{2} \varphi_{0,0}\left(t_{2}\right) d t_{2} \\
= & \frac{3^{3}}{2^{6}} \\
\text { Then, } c_{0,0 ; 0,0}^{2}= & \frac{3^{6}}{2^{12}}
\end{aligned}
$$

To evaluate $\sum_{j=1}^{\infty} d^{\prime 2}{ }_{j, 0 ; 0,0}$

$$
\begin{aligned}
d_{j, 0 ; 0,0}^{\prime}= & <f, \psi_{j, 0 ; 0,0}^{\prime}> \\
= & \int_{0}^{1 / 2^{j}} \int_{0}^{3 / 4}\left(t_{1}+t_{2}\right) \psi_{j, 0}\left(t_{1}\right) \varphi_{0,0}\left(t_{2}\right) d t_{1} d t_{2} \\
= & \int_{0}^{1 / 2^{j}} t_{1} \psi_{j, 0}\left(t_{1}\right) d t_{1} \int_{0}^{3 / 4} \varphi_{0,0}\left(t_{2}\right) d t_{2} \\
& +\int_{0}^{1 / 2^{j}} \psi_{j, 0}\left(t_{1}\right) d t_{1} \int_{0}^{3 / 4} t_{2} \varphi_{0,0}\left(t_{2}\right) d t_{2} \\
= & -\frac{3}{2^{4}} \frac{1}{2^{3 j / 2}} \\
\text { Then, } \sum_{j=1}^{\infty}{d^{\prime}}_{j, 0 ; 0,0}^{2}= & \sum_{j=1}^{\infty}\left(-\frac{3}{2^{4}} \frac{1}{2^{3 j / 2}}\right)^{2}=\frac{9}{2^{8}}\left(\sum_{j=1}^{\infty} \frac{1}{2^{3 j}}\right) \\
= & \frac{9}{2^{8}}\left(\frac{1 / 8}{1-1 / 8}\right)=\frac{9}{7 * 2^{8}}
\end{aligned}
$$

To evaluate $\sum_{j=2}^{\infty} \sum_{k=1}^{3 * 2^{j-2}-1} d^{\prime 2}{ }_{j, k ; 0,0}$

$$
\begin{aligned}
& d_{j, k ; 0,0}^{\prime}=<f, \psi_{j, k ; 0,0}^{\prime}> \\
&= \int_{k / 2^{j}}^{(k+1) / 2^{j}} \int_{0}^{3 / 4}\left(t_{1}+t_{2}\right) \\
& * \psi_{j, k}\left(t_{1}\right) \varphi_{0,0}\left(t_{2}\right) d t_{1} d t_{2} \\
&= \int_{k / 2^{j}}^{(k+1) / 2^{j}} t_{1} \psi_{j, k}\left(t_{1}\right) d t_{1} \\
& \int_{0}^{3 / 4} \varphi_{0,0}\left(t_{2}\right) d t_{2} \\
&+\int_{k / 2^{j}}^{(k+1) / 2^{j}} \psi_{j, k}\left(t_{1}\right) d t_{1} \\
& \int_{0}^{3 / 4} t_{2} \varphi_{0,0}\left(t_{2}\right) d t_{2} \\
&=-\frac{3}{2^{4}} \frac{1}{2^{3 j / 2}} \\
& d_{j, k ; 0,0}^{\prime 2} \sum_{j=2}^{\infty} \sum_{k=1}^{3 * 2-2}\left(-\frac{3}{2^{4}} \frac{1}{2^{3 j / 2}}\right)^{2} \\
&= \frac{9}{2^{8}} \sum_{j=2}^{\infty} \frac{1}{2^{3 j}}\left(\sum_{k=1}^{3 * 2^{j-2}-1} 1\right) \\
&= \frac{9}{2^{8}} \sum_{j=2}^{\infty}\left(\frac{3}{4} \frac{1}{2^{2 j}}-\frac{1}{2^{3 j}}\right) \\
&= \frac{9}{2^{8}}\left\{\frac{3}{4}\left(\sum_{j=2}^{\infty} \frac{1}{2^{2 j}}\right)-\left(\sum_{j=2}^{\infty} \frac{1}{2^{3 j}}\right)\right\} \\
&\left.\left.\frac{25}{1-1 / 2^{4}}\right)-\left(\frac{1 / 2^{6}}{1-1 / 2^{3}}\right)\right\} \\
&=
\end{aligned}
$$$$
\text { Then, } \sum_{j=2}^{\infty} \sum_{k=1}^{3 * 2^{j-2}-1} d^{\prime 2}{ }_{j, k ; 0,0}=\sum_{j=2}^{\infty} \sum_{k=1}^{3 * 2^{j-2}-1}\left(-\frac{3}{2^{4}} \frac{1}{2^{3 j / 2}}\right)^{2}
$$

To evaluate $d_{0,0 ; 0,0}^{\prime 2}$

$$
\begin{aligned}
d_{0,0 ; 0,0}^{\prime}= & <f, \psi_{0,0 ; 0,0}^{\prime}> \\
= & \int_{0}^{3 / 4} \int_{0}^{3 / 4}\left(t_{1}+t_{2}\right) \psi_{0,0}\left(t_{1}\right) \varphi_{0,0}\left(t_{2}\right) d t_{1} d t_{2} \\
= & \int_{0}^{3 / 4} t_{1} \psi_{0,0}\left(t_{1}\right) d t_{1} \int_{0}^{3 / 4} \varphi_{0,0}\left(t_{2}\right) d t_{2} \\
& +\int_{0}^{3 / 4} \psi_{0,0}\left(t_{1}\right) d t_{1} \int_{0}^{3 / 4} t_{2} \varphi_{0,0}\left(t_{2}\right) d t_{2} \\
= & \frac{3}{2^{6}}
\end{aligned}
$$

Then, $d_{0,0 ; 0,0}^{\prime 2}=\frac{3^{2}}{2^{12}}$ 
To evaluate $d_{1,1 ; 0,0}^{\prime 2}$

$$
\begin{aligned}
d_{1,1 ; 0,0}^{\prime}= & <f, \psi_{1,1 ; 0,0}^{\prime}> \\
= & \int_{1 / 2}^{3 / 4} \int_{0}^{3 / 4}\left(t_{1}+t_{2}\right) \psi_{1,1}\left(t_{1}\right) \varphi_{0,0}\left(t_{2}\right) d t_{1} d t_{2} \\
= & \int_{1 / 2}^{3 / 4} t_{1} \psi_{1,1}\left(t_{1}\right) d t_{1} \int_{0}^{3 / 4} \varphi_{0,0}\left(t_{2}\right) d t_{2} \\
& +\int_{1 / 2}^{3 / 4} \psi_{1,1}\left(t_{1}\right) d t_{1} \int_{0}^{3 / 4} t_{2} \varphi_{0,0}\left(t_{2}\right) d t_{2} \\
= & \frac{3}{2^{7 / 2}}
\end{aligned}
$$

Then, $d_{1,1 ; 0,0}^{\prime 2}=\frac{3^{2}}{2^{7}}$

To evaluate $\sum_{j^{\prime}=1}^{\infty} c_{0,0 ; j^{\prime}, 0}^{2}$

$$
\begin{aligned}
& \because \sum_{j=1}^{\infty}{d^{\prime}}_{j, 0 ; 0,0}^{2}=\frac{9}{7 * 2^{8}} \\
& \therefore \sum_{j^{\prime}=1}^{\infty}{c^{\prime}}_{0,0 ; j^{\prime}, 0}^{2}=\frac{9}{7 * 2^{8}}
\end{aligned}
$$

To evaluate $\sum_{j^{\prime}=2}^{\infty} \sum_{k^{\prime}=1}^{3 * 2^{j^{\prime}}-2}-1 c_{0,0 ; j^{\prime}, k^{\prime}}^{\prime 2}$

$$
\begin{aligned}
& \because \sum_{j=2}^{\infty} \sum_{k=1}^{3 * 2^{j-2}-1} d_{j, k ; 0,0}^{\prime 2}=\frac{45}{7 * 2^{12}} \\
& \therefore \sum_{j^{\prime}=2}^{\infty} \sum_{k^{\prime}=1}^{3 * 2^{j^{\prime}-2}-1} c_{0,0 ; j^{\prime}, k^{\prime}}^{\prime 2}=\frac{45}{7 * 2^{12}}
\end{aligned}
$$

To evaluate ${c^{\prime 2}}_{0,0 ; 0,0}^{2}$

$$
\begin{aligned}
& \because d_{0,0 ; 0,0}^{2}=\frac{3^{2}}{2^{12}} \\
& \therefore c_{0,0 ; 0,0}^{\prime 2}=\frac{3^{2}}{2^{12}}
\end{aligned}
$$

To evaluate ${c^{\prime}}_{0,0 ; 1,1}^{2}$

$$
\begin{aligned}
& \because d_{1,1 ; 0,0}^{2}=\frac{3^{2}}{2^{7}} \\
& \therefore c_{0,0 ; 1,1}^{\prime 2}=\frac{3^{2}}{2^{7}}
\end{aligned}
$$

To evaluate $\sum_{j=1}^{\infty} \sum_{j^{\prime}=1}^{\infty} d_{j, 0 ; j^{\prime}, 0}^{2}$

$$
\begin{aligned}
d_{j, 0 ; j^{\prime}, 0}= & <f, \psi_{j, 0 ; j^{\prime}, 0}> \\
= & \int_{0}^{1 / 2^{j}} \int_{0}^{1 / 2^{j^{\prime}}}\left(t_{1}+t_{2}\right) \psi_{j, 0}\left(t_{1}\right) \\
& * \psi_{j^{\prime}, 0}\left(t_{2}\right) d t_{1} d t_{2} \\
= & \int_{0}^{1 / 2^{j}} t_{1} \psi_{j, 0}\left(t_{1}\right) d t_{1} \\
& \int_{0}^{1 / 2^{j^{\prime}}} \psi_{j^{\prime}, 0}\left(t_{2}\right) d t_{2} \\
& +\int_{0}^{1 / 2^{j}} \psi_{j, 0}\left(t_{1}\right) d t_{1} \\
& \int_{0}^{1 / 2^{j^{\prime}}} t_{2} \psi_{j^{\prime}, 0}\left(t_{2}\right) d t_{2} \\
= & 0
\end{aligned}
$$

Then, $\sum_{j=1}^{\infty} \sum_{j^{\prime}=1}^{\infty} d_{j, 0 ; j^{\prime}, 0}^{2}=0$

To evaluate $\sum_{j=2}^{\infty} \sum_{k=1}^{3 * 2^{j-2}-1} \sum_{j^{\prime}=1}^{\infty} d_{j, k ; j^{\prime}, 0}^{2}$

$$
\begin{aligned}
d_{j, k ; j^{\prime}, 0}= & <f, \psi_{j, k ; j^{\prime}, 0}> \\
= & \int_{k / 2^{j}}^{(k+1) / 2^{j}} \int_{0}^{1 / 2^{j^{\prime}}}\left(t_{1}+t_{2}\right) \\
& * \psi_{j, k}\left(t_{1}\right) \psi_{j^{\prime}, 0}\left(t_{2}\right) d t_{1} d t_{2} \\
= & \int_{k / 2^{j}}^{(k+1) / 2^{j}} t_{1} \psi_{j, k}\left(t_{1}\right) d t_{1} \\
& \int_{0}^{1 / 2^{j^{\prime}}} \psi_{j^{\prime}, 0}\left(t_{2}\right) d t_{2} \\
& +\int_{k / 2^{j}}^{(k+1) / 2^{j}} \psi_{j, k}\left(t_{1}\right) d t_{1} \\
& \int_{0}^{1 / 2^{j^{\prime}}} t_{2} \psi_{j^{\prime}, 0}\left(t_{2}\right) d t_{2} \\
= & 0
\end{aligned}
$$

Then, $\sum_{j=2}^{\infty} \sum_{k=1}^{3 * 2^{j-2}-1} \sum_{j^{\prime}=1}^{\infty} d_{j, k ; j^{\prime}, 0}^{2}=0$

To evaluate $\sum_{j^{\prime}=1}^{\infty} d_{0,0 ; j^{\prime}, 0}^{2}$

$$
\begin{aligned}
d_{0,0 ; j^{\prime}, 0}= & <f, \psi_{0,0 ; j^{\prime}, 0}> \\
= & \int_{0}^{3 / 4} \int_{0}^{1 / 2^{j^{\prime}}}\left(t_{1}+t_{2}\right) \\
& * \psi_{0,0}\left(t_{1}\right) \psi_{j^{\prime}, 0}\left(t_{2}\right) d t_{1} d t_{2} \\
= & \int_{0}^{3 / 4} t_{1} \psi_{0,0}\left(t_{1}\right) d t_{1} \\
& \int_{0}^{1 / 2^{j^{\prime}}} \psi_{j^{\prime}, 0}\left(t_{2}\right) d t_{2} \\
& +\int_{0}^{3 / 4} \psi_{0,0}\left(t_{1}\right) d t_{1}
\end{aligned}
$$




$$
\begin{aligned}
& \int_{0}^{1 / 2^{j^{\prime}}} t_{2} \psi_{j^{\prime}, 0}\left(t_{2}\right) d t_{2} \\
= & -\frac{1}{2^{4}} \frac{1}{2^{3 j^{\prime} / 2}}
\end{aligned}
$$

Then, $\sum_{j^{\prime}=1}^{\infty} d_{0,0 ; j^{\prime}, 0}^{2}=\sum_{j^{\prime}=1}^{\infty}\left(-\frac{1}{2^{4}} \frac{1}{2^{3 j^{\prime} / 2}}\right)$

$$
\begin{aligned}
& =\frac{1}{2^{8}}\left(\sum_{j^{\prime}=1}^{\infty} \frac{1}{2^{3 j^{\prime}}}\right) \\
& =\frac{1}{2^{8}}\left(\frac{1 / 2^{3}}{1-1 / 2^{3}}\right)=\frac{1}{7 * 2^{8}}
\end{aligned}
$$

To evaluate $\sum_{j^{\prime}=1}^{\infty} d_{1,1 ; j^{\prime}, 0}^{2}$

$$
\begin{aligned}
d_{1,1 ; j^{\prime}, 0}= & <f, \psi_{1,1 ; j^{\prime}, 0}> \\
= & \int_{1 / 2}^{3 / 4} \int_{0}^{1 / 2^{j^{\prime}}}\left(t_{1}+t_{2}\right) \psi_{1,1}\left(t_{1}\right) \\
& * \psi_{j^{\prime}, 0}\left(t_{2}\right) d t_{1} d t_{2} \\
= & \int_{1 / 2}^{3 / 4} t_{1} \psi_{1,1}\left(t_{1}\right) d t_{1} \int_{0}^{1 / 2^{j^{\prime}}} \psi_{j^{\prime}, 0}\left(t_{2}\right) d t_{2} \\
+ & \int_{1 / 2}^{3 / 4} \psi_{1,1}\left(t_{1}\right) d t_{1} \int_{0}^{1 / 2^{j^{\prime}}} t_{2} \psi_{j^{\prime}, 0}\left(t_{2}\right) d t_{2} \\
= & -\frac{1}{2^{7 / 2}} \frac{1}{2^{3 j^{\prime} / 2}}
\end{aligned}
$$

Then, $\sum_{j^{\prime}=1}^{\infty} d_{1,1 ; j^{\prime}, 0}^{2}=\sum_{j^{\prime}=1}^{\infty}\left(-\frac{1}{2^{7 / 2}} \frac{1}{2^{3 j^{\prime} / 2}}\right)=\frac{1}{2^{7}}\left(\sum_{j^{\prime}=1}^{\infty} \frac{1}{2^{3 j^{\prime}}}\right)$

$$
=\frac{1}{2^{7}}\left(\frac{1 / 2^{3}}{1-1 / 2^{3}}\right)=\frac{1}{7 * 2^{7}}
$$

To evaluate $\sum_{j=1}^{\infty} \sum_{j^{\prime}=2}^{\infty} \sum_{k^{\prime}=1}^{3 * 2^{j^{\prime}-2}-1} d_{j, 0 ; j^{\prime}, k^{\prime}}^{2}$

$$
\begin{aligned}
& \because \sum_{j=2}^{\infty} \sum_{k=1}^{3 * 2^{j-2}-1} \sum_{j^{\prime}=1}^{\infty} d_{j, k ; j^{\prime}, 0}^{2}=0 \\
& \therefore \sum_{j=1}^{\infty} \sum_{j^{\prime}=2}^{\infty} \sum_{k^{\prime}=1}^{3 * 2 j^{j^{\prime}-2}-1} d_{j, 0 ; j^{\prime}, k^{\prime}}^{2}=0
\end{aligned}
$$

To evaluate $\sum_{j=2}^{\infty} \sum_{k=1}^{3 * 2^{j-2}-1} \sum_{j^{\prime}=2}^{\infty} \sum_{k^{\prime}=1}^{3 * 2^{j^{\prime}-2}-1} d_{j, k ; j^{\prime}, k^{\prime}}^{2}$

$$
\begin{aligned}
d_{j, k ; j^{\prime}, k^{\prime}}= & <f, \psi_{j, k ; j^{\prime}, k^{\prime}}> \\
= & \int_{k / 2^{j}}^{(k+1) / 2^{j}} \int_{k^{\prime} / 2^{j^{\prime}}}^{\left(k^{\prime}+1\right) / 2^{j^{\prime}}}\left(t_{1}+t_{2}\right) \\
& * \psi_{j, k}\left(t_{1}\right) \psi_{j^{\prime}, k^{\prime}}\left(t_{2}\right) d t_{1} d t_{2} \\
= & \int_{k / 2^{j}}^{(k+1) / 2^{j}} t_{1} \psi_{j, k}\left(t_{1}\right) d t_{1} \int_{k^{\prime} / 2^{j^{\prime}}}^{\left(k^{\prime}+1\right) / 2^{j^{\prime}}} \psi_{j^{\prime}, k^{\prime}}\left(t_{2}\right) d t_{2} \\
+ & \int_{k / 2^{j}}^{(k+1) / 2^{j}} \psi_{j, k}\left(t_{1}\right) d t_{1} \int_{k^{\prime} / 2^{j^{\prime}}}^{\left(k^{\prime}+1\right) / 2^{j^{\prime}}} t_{2} \psi_{j^{\prime}, k^{\prime}}\left(t_{2}\right) d t_{2} \\
= & 0
\end{aligned}
$$

$$
\text { Then, } \sum_{j=2}^{\infty} \sum_{k=1}^{3 * 2^{j-2}} \sum_{j^{\prime}=2}^{\infty} \sum_{k^{\prime}=1}^{3 * 2^{j^{\prime}-2}-1} d_{j, k ; j^{\prime}, k^{\prime}}^{2}=0
$$

To evaluate $\sum_{j^{\prime}=2}^{\infty} \sum_{k^{\prime}=1}^{3 * 2^{j^{\prime}-2}-1} d_{0,0 ; j^{\prime}, k^{\prime}}^{2}$

$$
\begin{aligned}
& d_{0,0 ; j^{\prime}, k^{\prime}}=<f, \psi_{0,0 ; j^{\prime}, k^{\prime}}> \\
& =\int_{0}^{3 / 4} \int_{k^{\prime} / 2^{j^{\prime}}}^{\left(k^{\prime}+1\right) / 2^{j^{\prime}}}\left(t_{1}+t_{2}\right) \psi_{0,0}\left(t_{1}\right) \psi_{j^{\prime}, k^{\prime}}\left(t_{2}\right) d t_{1} d t_{2} \\
& =\int_{0}^{3 / 4} t_{1} \psi_{0,0}\left(t_{1}\right) d t_{1} \int_{k^{\prime} / 2^{j^{\prime}}}^{\left(k^{\prime}+1\right) / 2^{j^{\prime}}} \psi_{j^{\prime}, k^{\prime}}\left(t_{2}\right) d t_{2} \\
& +\int_{0}^{3 / 4} \psi_{0,0}\left(t_{1}\right) d t_{1} \int_{k^{\prime} / 2^{j^{\prime}}}^{\left(k^{\prime}+1\right) / 2^{j^{\prime}}} t_{2} \psi_{j^{\prime}, k^{\prime}}\left(t_{2}\right) d t_{2} \\
& =-\frac{1}{2^{4} * 2^{3 j^{\prime} / 2}} \\
& =\frac{1}{2^{8}} \sum_{j^{\prime}=2}^{\infty} \frac{1}{2^{3 j^{\prime}}}\left(\sum_{k^{\prime}=1}^{3 * 2^{j^{\prime}-2}-1} 1\right) \\
& =\frac{1}{2^{8}}\left\{\frac{3}{4}\left(\sum_{j^{\prime}=2}^{\infty} \frac{1}{2^{2 j^{\prime}}}\right)-\left(\sum_{j^{\prime}=2}^{\infty} \frac{1}{2^{3 j^{\prime}}}\right)\right\} \\
& =\frac{1}{2^{8}}\left\{\frac{3}{4}\left(\frac{1 / 2^{4}}{1-1 / 2^{2}}\right)-\left(\frac{1 / 2^{6}}{1-1 / 2^{3}}\right)\right\} \\
& =\frac{5}{7 * 2^{12}}
\end{aligned}
$$

To evaluate $\sum_{j^{\prime}=2}^{\infty} \sum_{k^{\prime}=1}^{3 * 2^{j^{\prime}-2}-1} d_{1,1 ; j^{\prime}, k^{\prime}}^{2}$

$$
\begin{aligned}
d_{1,1 ; j^{\prime}, k^{\prime}}= & <f, \psi_{1,1 ; j^{\prime}, k^{\prime}}> \\
= & \int_{1 / 2}^{3 / 4} \int_{k^{\prime} / 2^{j^{\prime}}}^{\left(k^{\prime}+1\right) / 2^{j^{\prime}}}\left(t_{1}+t_{2}\right) \psi_{1,1}\left(t_{1}\right) \psi_{j^{\prime}, k^{\prime}}\left(t_{2}\right) d t_{1} d t_{2} \\
= & \int_{1 / 2}^{3 / 4} t_{1} \psi_{1,1}\left(t_{1}\right) d t_{1} \int_{k^{\prime} / 2^{j^{\prime}}}^{\left(k^{\prime}+1\right) / 2^{j^{\prime}}} \psi_{j^{\prime}, k^{\prime}}\left(t_{2}\right) d t_{2} \\
& +\int_{1 / 2}^{3 / 4} \psi_{1,1}\left(t_{1}\right) d t_{1} \int_{k^{\prime} / 2^{j^{\prime}}}^{\left(k^{\prime}+1\right) / 2^{j^{\prime}}} t_{2} \psi_{j^{\prime}, k^{\prime}}\left(t_{2}\right) d t_{2} \\
= & -\frac{1}{2^{7 / 2} * 2^{3 j^{\prime} / 2}}
\end{aligned}
$$


Then, $\sum_{j^{\prime}=2}^{\infty} \sum_{k^{\prime}=1}^{3 * 2^{j^{\prime}-2}-1} d_{1,1 ; j^{\prime}, k^{\prime}}^{2}=\sum_{j^{\prime}=2}^{\infty} \sum_{k^{\prime}=1}^{3 * 2^{j^{\prime}-2}-1}\left(-\frac{1}{2^{7 / 2} * 2^{3 j^{\prime} / 2}}\right)^{2}$

$$
\begin{aligned}
& =\frac{1}{2^{7}} \sum_{j^{\prime}=2}^{\infty} \frac{1}{2^{3 j^{\prime}}}\left(\sum_{k^{\prime}=1}^{3 * 2^{j^{\prime}-2}-1} 1\right) \\
& =\frac{1}{2^{7}}\left\{\frac{3}{4}\left(\sum_{j^{\prime}=2}^{\infty} \frac{1}{2^{2 j^{\prime}}}\right)-\left(\sum_{j^{\prime}=2}^{\infty} \frac{1}{2^{3 j^{\prime}}}\right)\right\} \\
& =\frac{1}{2^{7}}\left\{\frac{3}{4}\left(\frac{1 / 2^{4}}{1-2^{2}}\right)-\left(\frac{1 / 2^{6}}{1-1 / 2^{3}}\right)\right\} \\
& =\frac{5}{7 * 2^{11}}
\end{aligned}
$$

To evaluate $\sum_{j=1}^{\infty} d_{j, 0 ; 0,0}^{2}$

$$
\begin{aligned}
& \because \sum_{j^{\prime}=1}^{\infty} d_{0,0 ; j^{\prime}, 0}^{2}=\frac{1}{7 * 2^{8}} \\
& \therefore \sum_{j=1}^{\infty} d_{j, 0 ; 0,0}^{2}=\frac{1}{7 * 2^{8}}
\end{aligned}
$$

To evaluate $\sum_{j=2}^{\infty} \sum_{k=1}^{3 * 2^{j-2}-1} d_{j, k ; 0,0}^{2}$

$$
\begin{aligned}
& \because \sum_{j^{\prime}=2}^{\infty} \sum_{k^{\prime}=1}^{3 * 2^{j^{\prime}-2}-1} d_{0,0 ; j^{\prime}, k^{\prime}}^{2}=\frac{5}{7 * 2^{12}} \\
& \therefore \sum_{j=2}^{\infty} \sum_{k=1}^{3 * 2^{j-2}-1} d_{j, k ; 0,0}^{2}=\frac{5}{7 * 2^{12}}
\end{aligned}
$$

To evaluate $d_{0,0 ; 0,0}^{2}$

$$
\begin{aligned}
d_{0,0 ; 0,0} & =<f, \psi_{0,0 ; 0,0}> \\
& =\int_{0}^{3 / 4} \int_{0}^{3 / 4}\left(t_{1}+t_{2}\right) \psi_{0,0}\left(t_{1}\right) \psi_{0,0}\left(t_{2}\right) d t_{1} d t_{2} \\
& =\int_{0}^{3 / 4} t_{1} \psi_{0,0}\left(t_{1}\right) d t_{1} \int_{0}^{3 / 4} \psi_{0,0}\left(t_{2}\right) d t_{2} \\
& +\int_{0}^{3 / 4} \psi_{0,0}\left(t_{1}\right) d t_{1} \int_{0}^{3 / 4} t_{2} \psi_{0,0}\left(t_{2}\right) d t_{2} \\
& =-\frac{1}{2^{6}}
\end{aligned}
$$

Then, $d_{0,0 ; 0,0}^{2}=\frac{1}{2^{12}}$

To evaluate $d_{1,1 ; 0,0}^{2}$

$$
\begin{aligned}
d_{1,1 ; 0,0} & =<f, \psi_{1,1 ; 0,0}> \\
& =\int_{1 / 2}^{3 / 4} \int_{0}^{3 / 4}\left(t_{1}+t_{2}\right) \psi_{1,1}\left(t_{1}\right) \psi_{0,0}\left(t_{2}\right) d t_{1} d t_{2} \\
& =\int_{1 / 2}^{3 / 4} t_{1} \psi_{1,1}\left(t_{1}\right) d t_{1} \int_{0}^{3 / 4} \psi_{0,0}\left(t_{2}\right) d t_{2} \\
& +\int_{1 / 2}^{3 / 4} \psi_{1,1}\left(t_{1}\right) d t_{1} \int_{0}^{3 / 4} t_{2} \psi_{0,0}\left(t_{2}\right) d t_{2} \\
& =\frac{1}{2^{9 / 2}}
\end{aligned}
$$

Then, $d_{1,1 ; 0,0}^{2}=\frac{1}{2^{9}}$

To evaluate $\sum_{j=1}^{\infty} d_{j, 0 ; 1,1}^{2}$

$$
\begin{aligned}
& \because \sum_{j^{\prime}=1}^{\infty} d_{1,1 ; j^{\prime}, 0}^{2}=\frac{1}{7 * 2^{7}} \\
& \therefore \sum_{j=1}^{\infty} d_{j, 0 ; 1,1}^{2}=\frac{1}{7 * 2^{7}}
\end{aligned}
$$

To evaluate $\sum_{j=2}^{\infty} \sum_{k=1}^{3 * 2^{j-2}-1} d_{j, k ; 1,1}^{2}$

$$
\begin{aligned}
& \because \sum_{j^{\prime}=2}^{\infty} \sum_{k^{\prime}=1}^{3 * 2^{j^{\prime}-2}-1} d_{1,1 ; j^{\prime}, k^{\prime}}^{2}=\frac{5}{7 * 2^{11}} \\
& \therefore \sum_{j=2}^{\infty} \sum_{k=1}^{3 * 2^{j-2}-1} d_{j, k ; 1,1}^{2}=\frac{5}{7 * 2^{11}}
\end{aligned}
$$

To evaluate $d_{0,0 ; 1,1}^{2}$

$$
\begin{aligned}
& \because d_{1,1 ; 0,0}^{2}=\frac{1}{2^{9}} \\
& \therefore d_{0,0 ; 1,1}^{2}=\frac{1}{2^{9}}
\end{aligned}
$$

To evaluate $d_{1,1 ; 1,1}^{2}$

$$
\begin{aligned}
d_{1,1 ; 1,1} & =<f, \psi_{1,1 ; 1,1}> \\
& =\int_{1 / 2}^{3 / 4} \int_{1 / 2}^{3 / 4}\left(t_{1}+t_{2}\right) \psi_{1,1}\left(t_{1}\right) \psi_{1,1}\left(t_{2}\right) d t_{1} d t_{2} \\
& =\int_{1 / 2}^{3 / 4} t_{1} \psi_{1,1}\left(t_{1}\right) d t_{1} \int_{1 / 2}^{3 / 4} \psi_{1,1}\left(t_{2}\right) d t_{2} \\
& +\int_{1 / 2}^{3 / 4} \psi_{1,1}\left(t_{1}\right) d t_{1} \int_{1 / 2}^{3 / 4} t_{2} \psi_{1,1}\left(t_{2}\right) d t_{2} \\
& =\frac{5}{2^{5}}
\end{aligned}
$$

Then, $d_{1,1 ; 1,1}^{2}=\frac{5^{2}}{2^{10}}$

putting these calculated values in equation $(35$, we get

$$
\begin{aligned}
\text { R.H.S }= & \frac{3^{6}}{2^{12}}+\frac{3^{2}}{2^{12}}+\frac{3^{2}}{2^{7}}+\frac{9}{7 * 2^{8}}+\frac{45}{7 * 2^{12}}+\frac{3^{2}}{2^{12}} \\
& +\frac{1}{2^{12}}+\frac{1}{2^{9}}+\frac{1}{7 * 2^{8}}+\frac{5}{7 * 2^{12}}+\frac{3^{2}}{2^{7}}+\frac{1}{2^{9}} \\
& +\frac{5^{2}}{2^{10}}+\frac{1}{7 * 2^{7}}+\frac{5}{7 * 2^{11}}+\frac{9}{7 * 2^{8}}+\frac{1}{7 * 2^{8}} \\
& +\frac{1}{7 * 2^{7}}+0+0+\frac{45}{7 * 2^{12}}+\frac{5}{7 * 2^{12}}+\frac{5}{7 * 2^{11}} \\
& +0+0 \\
= & \frac{189}{2^{9}}
\end{aligned}
$$

By equation (34) and (36, we get

L.H.S. = R.H.S. 


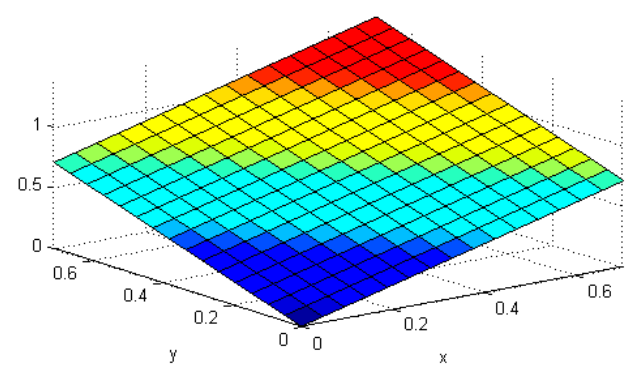

Fig. 17. Graph of signal $f\left(t_{1}, t_{2}\right)=t_{1}+t_{2}, t \in[0,3 / 4) \times[0,3 / 4)$ with order of matrix $=16$

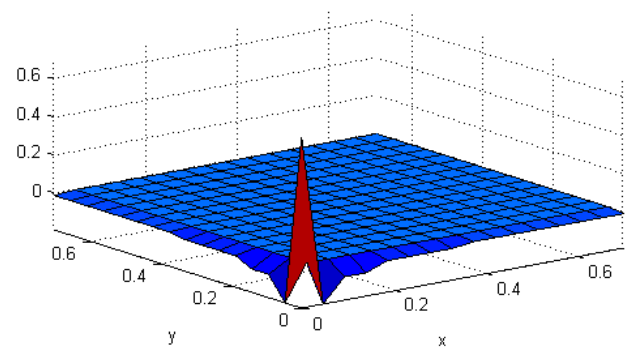

Fig. 18. Graph of decomposed signal $f\left(t_{1}, t_{2}\right)=t_{1}+t_{2}, t \in[0,1) \times$ $[0,1)$ with order of matrix $=16$ at resolutions $m_{1}=m_{2}=0$

7.9 Expansion of signal $f\left(t_{1}, t_{2}\right)=\chi_{[0,1]}(t) \chi_{[0,1]}(t)$, at resolutions $m$ and $m^{\prime}$.

From equation (4), double Haar wavelet series for a signal $f \in$ $L^{2}(\mathbb{R} \times \mathbb{R})$ in the region $[0,1) \times[0,1)$ at resolutions $m$ and $m^{\prime}$ is given by,

$$
\begin{aligned}
& f\left(t_{1}, t_{2}\right)=\sum_{n=0}^{2^{m}-1} \sum_{n^{\prime}=0}^{2^{m^{\prime}}-1} c_{m, n ; m^{\prime}, n^{\prime}} \varphi_{m, n ; m^{\prime}, n^{\prime}}\left(t_{1}, t_{2}\right) \\
&+ \sum_{n=0}^{2^{m}-1} \sum_{j^{\prime}=m^{\prime}}^{\infty} \sum_{k^{\prime}=0}^{2^{j^{\prime}}-1} c_{m, n ; j^{\prime}, k^{\prime}}^{\prime} \varphi_{m, n ; j^{\prime}, k^{\prime}}^{\prime}\left(t_{1}, t_{2}\right) \\
&+\quad \sum_{j=m}^{\infty} \sum_{k=0}^{2^{j}-1} \sum_{n^{\prime}=0}^{m^{\prime}-1} d_{j, k ; m^{\prime}, n^{\prime}}^{\prime} \psi_{j, k ; m^{\prime}, n^{\prime}}^{\prime}\left(t_{1}, t_{2}\right) \\
&+\quad \sum_{j=m}^{\infty} \sum_{k=0}^{2^{j}-1} \sum_{j^{\prime}=m^{\prime}}^{\infty} \sum_{k^{\prime}=0}^{2^{j^{\prime}}-1} d_{j, k ; j^{\prime}, k^{\prime}} \psi_{j, k ; j^{\prime}, k^{\prime}}\left(t_{1}, t_{2}\right)
\end{aligned}
$$

To evaluate $c_{m, n ; m, n^{\prime}}$

$$
\begin{aligned}
c_{m, n ; m, n^{\prime}}= & <f, \varphi_{m, n ; m^{\prime}, n^{\prime}}> \\
= & \int_{n / 2^{m}}^{(n+1) / 2^{m}} \int_{n^{\prime} / 2^{m^{\prime}}}^{\left(n^{\prime}+1\right) / 2^{m^{\prime}}} \\
& \varphi_{m, n}\left(t_{1}\right) \varphi_{m^{\prime}, n^{\prime}}\left(t_{2}\right) d t_{1} d t_{2} \\
= & \int_{n / 2^{m}}^{(n+1) / 2^{m}} \varphi_{m, n}\left(t_{1}\right) d t_{1} \\
= & \frac{1}{2^{\left(m+m^{\prime}\right) / 2}}
\end{aligned}
$$

To evaluate $c_{m, n ; j^{\prime}, k^{\prime}}^{\prime}$

$$
\begin{aligned}
c_{m, n ; j^{\prime}, k^{\prime}}^{\prime}= & <f, \varphi_{m, n ; j^{\prime}, k^{\prime}}^{\prime}> \\
= & \int_{n / 2^{m}}^{(n+1) / 2^{m}} \int_{k^{\prime} / 2^{j^{\prime}}}^{\left(k^{\prime}+1\right) / 2^{j^{\prime}}} \\
& \varphi_{m, n}\left(t_{1}\right) \psi_{j^{\prime}, k^{\prime}}\left(t_{2}\right) d t_{1} d t_{2} \\
= & 0
\end{aligned}
$$

To evaluate $d_{j, k ; m^{\prime}, n^{\prime}}^{\prime}$

$$
\begin{aligned}
d_{j, k ; m^{\prime}, n^{\prime}}^{\prime} & =<f, \psi_{j, k ; m^{\prime}, n^{\prime}}^{\prime}> \\
= & \int_{k / 2^{j}}^{(k+1) / 2^{j}} \int_{n^{\prime} / 2^{m^{\prime}}}^{\left(n^{\prime}+1\right) / 2^{m^{\prime}}} \\
& \psi_{j, k}\left(t_{1}\right) \varphi_{m^{\prime}, n^{\prime}}\left(t_{2}\right) d t_{1} d t_{2} \\
= & 0
\end{aligned}
$$

To evaluate $d_{j, k ; j^{\prime}, k^{\prime}}$

$$
\begin{aligned}
d_{j, k ; j^{\prime}, k^{\prime}}= & <f, \psi_{j, k ; j^{\prime}, k^{\prime}}> \\
= & \int_{k / 2^{j}}^{(k+1) / 2^{j}} \int_{k^{\prime} / 2^{j^{\prime}}}^{\left(k^{\prime}+1\right) / 2^{j^{\prime}}} \\
& \psi_{j, k}\left(t_{1}\right) \psi_{j^{\prime}, k^{\prime}}\left(t_{2}\right) d t_{1} d t_{2} \\
= & 0
\end{aligned}
$$

taking square of norm of both sides of equation 30

$$
\begin{aligned}
& \|f\|_{2}^{2}=\sum_{n=0}^{2^{m}-1} \sum_{n^{\prime}=0}^{2^{m^{\prime}}-1} c_{m, n ; m^{\prime}, n^{\prime}}^{2} \\
& +\sum_{n=0}^{2^{m}-1} \sum_{j^{\prime}=m^{\prime}}^{\infty} \sum_{k^{\prime}=0}^{2^{j^{\prime}}-1} c_{m, n ; j^{\prime}, k^{\prime}}^{\prime 2} \\
& +\quad \sum_{j=m}^{\infty} \sum_{k=0}^{2^{j}-1} \sum_{n^{\prime}=0}^{2^{m^{\prime}}-1} d^{\prime 2}{ }_{j, k ; m^{\prime}, n^{\prime}} \\
& +\quad \sum_{j=m}^{\infty} \sum_{k=0}^{2^{j}-1} \sum_{j^{\prime}=m^{\prime}}^{\infty} \sum_{k^{\prime}=0}^{2^{j^{\prime}}-1} d_{j, k ; j^{\prime}, k^{\prime}}^{2} \\
& \text { L.H.S. }=\|f\|_{2}^{2}=\int_{0}^{1} \int_{0}^{1} d t_{1} d t_{2} \\
& =1
\end{aligned}
$$




$$
\begin{aligned}
R . H . S & =\sum_{n=0}^{2^{m}-1} \sum_{n^{\prime}=0}^{2^{m^{\prime}}-1} c_{m, n ; m^{\prime}, n^{\prime}}^{2} \\
& +\sum_{n=0}^{2^{m}-1} \sum_{j^{\prime}=m^{\prime}}^{\infty} \sum_{k^{\prime}=0}^{2^{j^{\prime}}-1}{c^{\prime}}_{m, n ; j^{\prime}, k^{\prime}}^{2} \\
& +\sum_{j=m}^{\infty} \sum_{k=0}^{2^{j}-1} \sum_{n^{\prime}=0}^{2^{m^{\prime}}-1} d_{j, k ; m^{\prime}, n^{\prime}}^{2} \\
& +\sum_{j=m}^{\infty} \sum_{k=0}^{2^{j}-1} \sum_{j^{\prime}=m^{\prime}}^{\infty} \sum_{k^{\prime}=0}^{2^{j^{\prime}}-1} d_{j, k ; j^{\prime}, k^{\prime}}^{2} \\
& =\sum_{n=0}^{2^{m}} \sum_{n^{\prime}=0}^{m^{m^{\prime}}-1} \frac{1}{2^{\left(m+m^{\prime}\right)}}+0+0+0 \\
& =1
\end{aligned}
$$

By equation (31) and (32), we get

$$
\text { L.H.S = R.H.S }
$$

Hence, double wavelet series is verified.

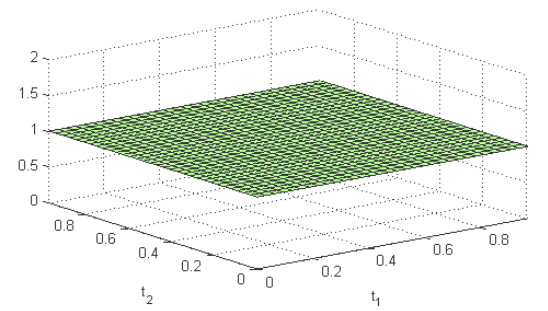

Fig. 19. Graph of signal $f\left(t_{1}, t_{2}\right)=\chi_{[0,1]}\left(t_{1}\right) \chi_{[0,1]}\left(t_{2}\right)$ with order of matrix $=16$

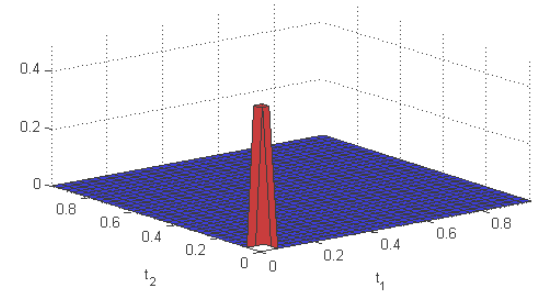

Fig. 20. Graph of decomposed signal $f\left(t_{1}, t_{2}\right)=\chi_{[0,1]}\left(t_{1}\right) \chi_{[0,1]}\left(t_{2}\right)$ with order of matrix $=16$ at resolutions $m_{1}=m_{2}=1$

\subsection{Expansion of signal}

$$
f\left(t_{1}, t_{2}\right)=\chi_{[0,3 / 4]}\left(t_{1}\right) \chi_{[0,3 / 4]}\left(t_{2}\right) \text {, at resolutions }
$$$$
m=m^{\prime}=0 \text {. }
$$

From equation ( 5 ), double Haar wavelet series for a signal $f \in$ $L^{2}(\mathbb{R} \times \mathbb{R})$ in the region $[0,3 / 4) \times[0.3 / 4)$ at resolutions $m=$ $m^{\prime}=0$ is given by,

$$
\begin{aligned}
& f\left(t_{1}, t_{2}\right)=c_{0,0 ; 0,0} \varphi_{0,0 ; 0,0}\left(t_{1}, t_{2}\right)+c_{0,0 ; 0,0}^{\prime} \varphi_{0,0 ; 0,0}^{\prime}\left(t_{1}, t_{2}\right) \\
& +c_{0,0 ; 1,1}^{\prime} \varphi_{0,0 ; 1,1}^{\prime}\left(t_{1}, t_{2}\right)+\sum_{j^{\prime}=1}^{\infty} c_{0,0 ; j^{\prime}, 0}^{\prime} \varphi_{0,0 ; j^{\prime}, 0}^{\prime}\left(t_{1}, t_{2}\right) \\
& +\sum_{j^{\prime}=2}^{\infty} \sum_{k^{\prime}=1}^{3 * j^{j^{\prime}-2}-1} c_{0,0 ; j^{\prime}, k^{\prime}}^{\prime} \varphi_{0,0 ; j^{\prime}, k^{\prime}}^{\prime}\left(t_{1}, t_{2}\right) \\
& +d_{0,0 ; 0,0}^{\prime} \psi_{0,0 ; 0,0}^{\prime}\left(t_{1}, t_{2}\right)+d_{0,0 ; 0,0} \psi_{0,0 ; 0,0}\left(t_{1}, t_{2}\right) \\
& +d_{0,0 ; 1,1} \psi_{0,0 ; 1,1}\left(t_{1}, t_{2}\right)+\sum_{j^{\prime}=1}^{\infty} d_{0,0 ; j^{\prime}, 0} \psi_{0,0 ; j^{\prime}, 0}\left(t_{1}, t_{2}\right) \\
& +\sum_{j^{\prime}=2}^{\infty} \sum_{k^{\prime}=1}^{3 * 2^{j^{\prime}-2}-1} d_{0,0 ; j^{\prime}, k^{\prime}} \psi_{0,0 ; j^{\prime}, k^{\prime}}\left(t_{1}, t_{2}\right) \\
& +d_{1,1 ; 0,0}^{\prime} \psi_{1,1 ; 0,0}^{\prime}\left(t_{1}, t_{2}\right)+d_{1,1 ; 0,0} \psi_{1,1 ; 0,0}\left(t_{1}, t_{2}\right) \\
& +d_{1,1 ; 1,1} \psi_{1,1 ; 1,1}\left(t_{1}, t_{2}\right)+\sum_{j^{\prime}=1}^{\infty} d_{1,1 ; j^{\prime}, 0} \psi_{1,1 ; j^{\prime}, 0}\left(t_{1}, t_{2}\right) \\
& +\sum_{j^{\prime}=2}^{\infty} \sum_{k^{\prime}=1}^{3 * 2 j^{j^{\prime}-2}-1} d_{1,1 ; j^{\prime} k^{\prime}} \psi_{1,1 ; j^{\prime}, k^{\prime}}\left(t_{1}, t_{2}\right) \\
& +\sum_{j=1}^{\infty} d_{j, 0 ; 0,0}^{\prime} \psi_{j, 0 ; 0,0}^{\prime}\left(t_{1}, t_{2}\right) \\
& +\sum_{j=1}^{\infty} d_{j, 0 ; 0,0} \psi_{j, 0 ; 0,0}\left(t_{1}, t_{2}\right)+\sum_{j=1}^{\infty} d_{j, 0 ; 1,1} \psi_{j, 0 ; 1,1}\left(t_{1}, t_{2}\right) \\
& +\sum_{j=1}^{\infty} \sum_{j^{\prime}=1}^{\infty} d_{j, 0 ; j^{\prime}, 0} \psi_{j, 0 ; j^{\prime}, 0}\left(t_{1}, t_{2}\right) \\
& +\sum_{j=1}^{\infty} \sum_{j^{\prime}=2}^{\infty} \sum_{k^{\prime}=1}^{3 * 2^{j^{\prime}-2}-1} d_{j, 0 ; j^{\prime}, k^{\prime}} \psi_{j, 0 ; j^{\prime}, k^{\prime}}\left(t_{1}, t_{2}\right) \\
& +\sum_{j=2}^{\infty} \sum_{k=1}^{3 * 2^{j-2}-1} d_{j, k ; 0,0}^{\prime} \psi_{j, k ; 0,0}^{\prime}\left(t_{1}, t_{2}\right) \\
& +\sum_{j=2}^{\infty} \sum_{k=1}^{3 * 2^{j-2}-1} d_{j, k ; 0,0} \psi_{j, k ; 0,0}\left(t_{1}, t_{2}\right) \\
& +\sum_{j=2}^{\infty} \sum_{k=1}^{3 * 2^{j-2}-1} d_{j, k ; 1,1} \psi_{j, k ; 1,1}\left(t_{1}, t_{2}\right) \\
& +\sum_{j=2}^{\infty} \sum_{k=1}^{3 * 2^{j-2}} \sum_{j^{\prime}=1}^{\infty} d_{j, k ; j^{\prime}, 0} \psi_{j, k ; j^{\prime}, 0}\left(t_{1}, t_{2}\right) \\
& +\sum_{j=2}^{\infty} \sum_{k=1}^{3 * 2^{j-2}-1} \sum_{j^{\prime}=2}^{\infty} \sum_{k^{\prime}=1}^{3 * j^{j^{\prime}-2}-1} d_{j, k ; j^{\prime}, k^{\prime}} \psi_{j, k ; j^{\prime}, k^{\prime}}\left(t_{1}, t_{2}\right)
\end{aligned}
$$


taking square of norm of both sides,

$$
\begin{aligned}
& \|f\|_{2}^{2}=c_{0,0 ; 0,0}^{2}\left\|\varphi_{0,0 ; 0,0}\right\|_{2}^{2} \\
& +c^{\prime 2}{ }_{0,0 ; 0,0}\left\|\varphi_{0,0 ; 0,0}^{\prime}\right\|_{2}^{2} \\
& +c_{0,0 ; 1,1}^{\prime 2}\left\|\varphi_{0,0 ; 1,1}^{\prime}\right\|_{2}^{2} \\
& +\sum_{j^{\prime}=1}^{\infty} c_{0,0 ; j^{\prime}, 0}^{\prime 2}\left\|\varphi_{0,0 ; j^{\prime}, 0}^{\prime}\right\|_{2}^{2} \\
& +\sum_{j^{\prime}=2}^{\infty} \sum_{k^{\prime}=1}^{3 * 2^{j^{\prime}-2}-1} c_{0,0 ; j^{\prime}, k^{\prime}}^{\prime 2}\left\|\varphi_{0,0 ; j^{\prime}, k^{\prime}}^{\prime}\right\|_{2}^{2} \\
& +d_{0,0 ; 0,0}^{\prime 2}\left\|\psi_{0,0 ; 0,0}^{\prime}\right\|_{2}^{2} \\
& +d_{0,0 ; 0,0}^{2}\left\|\psi_{0,0 ; 0,0}\right\|_{2}^{2} \\
& +d_{0,0 ; 1,1}^{2}\left\|\psi_{0,0 ; 1,1}\right\|_{2}^{2} \\
& +\sum_{j^{\prime}=1}^{\infty} d_{0,0 ; j^{\prime}, 0}^{2}\left\|\psi_{0,0 ; j^{\prime}, 0}\right\|_{2}^{2} \\
& +\sum_{j^{\prime}=2}^{\infty} \sum_{k^{\prime}=1}^{3 * 2^{j^{\prime}-2}-1} d_{0,0 ; j^{\prime}, k^{\prime}}^{2}\left\|\psi_{0,0 ; j^{\prime}, k^{\prime}}\right\|_{2}^{2} \\
& +d_{1,1 ; 0,0}^{\prime 2}\left\|\psi_{1,1 ; 0,0}^{\prime}\right\|_{2}^{2} \\
& +d_{1,1 ; 0,0}^{2}\left\|\psi_{1,1 ; 0,0}\right\|_{2}^{2} \\
& +d_{1,1 ; 1,1}^{2}\left\|\psi_{1,1 ; 1,1}\right\|_{2}^{2} \\
& +\sum_{j^{\prime}=1}^{\infty} d_{1,1 ; j^{\prime}, 0}^{2}\left\|\psi_{1,1 ; j^{\prime}, 0}\right\|_{2}^{2} \\
& +\sum_{j^{\prime}=2}^{\infty} \sum_{k^{\prime}=1}^{3 * 2^{j^{\prime}-2}-1} d_{1,1 ; j^{\prime} k^{\prime}}^{2}\left\|\psi_{1,1 ; j^{\prime}, k^{\prime}}\right\|_{2}^{2} \\
& +\sum_{j=1}^{\infty}{d^{\prime}}_{j, 0 ; 0,0}^{2}\left\|\psi_{j, 0 ; 0,0}^{\prime}\right\|_{2}^{2} \\
& +\sum_{j=1}^{\infty} d_{j, 0 ; 0,0}^{2}\left\|\psi_{j, 0 ; 0,0}\right\|_{2}^{2} \\
& +\sum_{j=1}^{\infty} d_{j, 0 ; 1,1}^{2}\left\|\psi_{j, 0 ; 1,1}\right\|_{2}^{2} \\
& +\sum_{j=1}^{\infty} \sum_{j^{\prime}=1}^{\infty} d_{j, 0 ; j^{\prime}, 0}^{2}\left\|\psi_{j, 0 ; j^{\prime}, 0}\right\|_{2}^{2} \\
& +\sum_{j=1}^{\infty} \sum_{j^{\prime}=2}^{\infty} \sum_{k^{\prime}=1}^{3 * 2^{j^{\prime}-2}-1} d_{j, 0 ; j^{\prime}, k^{\prime}}^{2}\left\|\psi_{j, 0 ; j^{\prime}, k^{\prime}}\right\|_{2}^{2} \\
& +\sum_{j=2}^{\infty} \sum_{k=1}^{3 * 2^{j-2}-1} d_{j, k ; 0,0}^{\prime 2}\left\|\psi_{j, k ; 0,0}^{\prime}\right\|_{2}^{2} \\
& +\sum_{j=2}^{\infty} \sum_{k=1}^{3 * 2^{j-2}-1} d_{j, k ; 0,0}^{2}\left\|\psi_{j, k ; 0,0}\right\|_{2}^{2} \\
& +\sum_{j=2}^{\infty} \sum_{k=1}^{3 * 2^{j-2}-1} d_{j, k ; 1,1}^{2}\left\|\psi_{j, k ; 1,1}\right\|_{2}^{2} \\
& +\sum_{j=2}^{\infty} \sum_{k=1}^{3 * 2^{j-2}-1} \sum_{j^{\prime}=1}^{\infty} d_{j, k ; j^{\prime}, 0}^{2}\left\|\psi_{j, k ; j^{\prime}, 0}\right\|_{2}^{2} \\
& +\sum_{j=2}^{\infty} \sum_{k=1}^{3 * 2^{j-2}} \sum_{j^{\prime}=2}^{\infty} \sum_{k^{\prime}=1}^{3 * j^{j^{\prime}-2}-1} d_{j, k ; j^{\prime}, k^{\prime}}^{2}\left\|\psi_{j, k ; j^{\prime}, k^{\prime}}\right\|_{2}^{2}
\end{aligned}
$$

$$
\begin{aligned}
& =c_{0,0 ; 0,0}^{2}+c_{0,0 ; 0,0}^{\prime 2}+c_{0,0 ; 1,1}^{\prime 2}+\sum_{j^{\prime}=1}^{\infty} c_{0,0 ; j^{\prime}, 0}^{\prime 2} \\
& +\sum_{j^{\prime}=2}^{\infty} \sum_{k^{\prime}=1}^{3 * 2 j^{j^{\prime}-2}-1} c_{0,0 ; j^{\prime}, k^{\prime}}^{\prime 2}+d_{0,0 ; 0,0}^{\prime 2}+d_{0,0 ; 0,0}^{2} \\
& +d_{0,0 ; 1,1}^{2}+\sum_{j^{\prime}=1}^{\infty} d_{0,0 ; j^{\prime}, 0}^{2}+\sum_{j^{\prime}=2}^{\infty} \sum_{k^{\prime}=1}^{3 * 2^{j^{\prime}}-2} d_{0,0 ; j^{\prime}, k^{\prime}}^{2} \\
& +d_{1,1 ; 0,0}^{2}+d_{1,1 ; 0,0}^{2}+d_{1,1 ; 1,1}^{2}+\sum_{j^{\prime}=1}^{\infty} d_{1,1 ; j^{\prime}, 0}^{2} \\
& +\sum_{j^{\prime}=2}^{\infty} \sum_{k^{\prime}=1}^{3 * 2^{j^{\prime}-2}-1} d_{1,1 ; j^{\prime}, k^{\prime}}^{2}+\sum_{j=1}^{\infty}{d^{\prime 2}}_{j, 0 ; 0,0}^{2}+\sum_{j=1}^{\infty} d_{j, 0 ; 0,0}^{2} \\
& +\sum_{j=1}^{\infty} d_{j, 0 ; 1,1}^{2}+\sum_{j=1}^{\infty} \sum_{j^{\prime}=1}^{\infty} d_{j, 0 ; j^{\prime}, 0}^{2}+\sum_{j=1}^{\infty} \sum_{j^{\prime}=2}^{\infty} \sum_{k^{\prime}=1}^{3 * 2^{j^{\prime}-2}-1} d_{j, 0 ; j^{\prime}, k^{\prime}}^{2} \\
& +\sum_{j=2}^{\infty} \sum_{k=1}^{3 * 2^{j-2}-1} d^{\prime 2}{ }_{j, k ; 0,0}+\sum_{j=2}^{\infty} \sum_{k=1}^{3 * 2^{j-2}-1} d_{j, k ; 0,0}^{2} \\
& +\sum_{j=2}^{\infty} \sum_{k=1}^{3 * 2^{j-2}-1} d_{j, k ; 1,1}^{2}+\sum_{j=2}^{\infty} \sum_{k=1}^{3 * 2^{j-2}-1} \sum_{j^{\prime}=1}^{\infty} d_{j, k ; j^{\prime}, 0}^{2} \\
& +\sum_{j=2}^{\infty} \sum_{k=1}^{3 * 2^{j-2}} \sum_{j^{\prime}=2}^{\infty} \sum_{k^{\prime}=1}^{3 * 2^{j^{\prime}-2}-1} d_{j, k ; j^{\prime}, k^{\prime}}^{2}
\end{aligned}
$$

$\because\left\|\varphi_{m, n ; m^{\prime}, n^{\prime}}\right\|_{2}^{2}=1,\left\|\varphi_{m, n ; j^{\prime}, k^{\prime}}^{\prime}\right\|_{2}^{2}=1,\left\|\psi_{j, k ; j^{\prime}, k^{\prime}}\right\|_{2}^{2}=1$, $\left\|\psi_{j, k ; m^{\prime}, n^{\prime}}^{\prime}\right\|_{2}^{2}=1$

taking L.H.S. of the equation,

$$
\begin{aligned}
& \text { L.H.S. }=\|f\|_{2}^{2}=\int_{0}^{3 / 4} \int_{0}^{3 / 4} d t_{1} d t_{2} \\
& =\frac{9}{2^{4}}
\end{aligned}
$$

$$
\begin{aligned}
R . H . S . & =c_{0,0 ; 0,0}^{2}+c_{0,0 ; 0,0}^{\prime 2}+c_{0,0 ; 1,1}^{\prime 2}+\sum_{j^{\prime}=1}^{\infty} c_{0,0 ; j^{\prime}, 0}^{2} \\
& +\sum_{j^{\prime}=2}^{\infty} \sum_{k^{\prime}=1}^{3 * 2^{j^{\prime}-2}-1} c_{0,0 ; j^{\prime}, k^{\prime}}^{\prime 2}+d^{\prime 2}{ }_{0,0 ; 0,0}+d_{0,0 ; 0,0}^{2} \\
& +d_{0,0 ; 1,1}^{2}+\sum_{j^{\prime}=1}^{\infty} d_{0,0 ; j^{\prime}, 0}^{2}+\sum_{j^{\prime}=2}^{\infty} \sum_{k^{\prime}=1}^{3 * 2 j^{j^{\prime}-2}-1} d_{0,0 ; j^{\prime}, k^{\prime}}^{2} \\
& +d_{1,1 ; 0,0}^{\prime 2}+d_{1,1 ; 0,0}^{2}+d_{1,1 ; 1,1}^{2}+\sum_{j^{\prime}=1}^{\infty} d_{1,1 ; j^{\prime}, 0}^{2} \\
& +\sum_{j^{\prime}=2}^{\infty} \sum_{k^{\prime}=1}^{3 * 2^{j^{\prime}-2}-1} d_{1,1 ; j^{\prime}, k^{\prime}}^{2}+\sum_{j=1}^{\infty} d_{j, 0 ; 0,0}^{\prime 2}+\sum_{j=1}^{\infty} d_{j, 0 ; 0,0}^{2} \\
& +\sum_{j=1}^{\infty} d_{j, 0 ; 1,1}^{2}+\sum_{j=1}^{\infty} \sum_{j^{\prime}=1}^{\infty} d_{j, 0 ; j^{\prime}, 0}^{2}
\end{aligned}
$$




$$
\begin{aligned}
& +\sum_{j=1}^{\infty} \sum_{j^{\prime}=2}^{\infty} \sum_{k^{\prime}=1}^{3 * 2^{j^{\prime}-2}-1} d_{j, 0 ; j^{\prime}, k^{\prime}}^{2}+\sum_{j=2}^{\infty} \sum_{k=1}^{3 * 2^{j-2}-1} d_{j, k ; 0,0}^{2} \\
& +\sum_{j=2}^{\infty} \sum_{k=1}^{3 * 2^{j-2}-1} d_{j, k ; 0,0}^{2}+\sum_{j=2}^{\infty} \sum_{k=1}^{3 * 2^{j-2}-1} d_{j, k ; 1,1}^{2} \\
& +\sum_{j=2}^{\infty} \sum_{k=1}^{3 * 2 \sum^{j-2}-1} \sum_{j^{\prime}=1}^{\infty} d_{j, k ; j^{\prime}, 0}^{2} \\
& +\sum_{j=2}^{\infty} \sum_{k=1}^{3 * 2^{j-2}-1} \sum_{j^{\prime}=2}^{\infty} \sum_{k^{\prime}=1}^{3 * j^{j^{\prime}-2}-1} d_{j, k ; j^{\prime}, k^{\prime}}^{2}
\end{aligned}
$$

we will calculate each term of this equation seprately with respect to the cases mentioned in table ( 17 .

To evaluate $c_{0,0 ; 0,0}^{2}$

$$
\begin{aligned}
c_{0,0 ; 0,0} & =<f, \varphi_{0,0 ; 0,0}> \\
& =\int_{0}^{3 / 4} \int_{0}^{3 / 4} \varphi_{0,0}\left(t_{1}\right) \varphi_{0,0}\left(t_{2}\right) d t_{1} d t_{2} \\
& =\int_{0}^{3 / 4} \varphi_{0,0}\left(t_{1}\right) d t_{1} \int_{0}^{3 / 4} \varphi_{0,0}\left(t_{2}\right) d t_{2} \\
& =\frac{9}{2^{4}}
\end{aligned}
$$$$
\text { Then, } c_{0,0 ; 0,0}^{2}=\frac{9^{2}}{2^{8}}
$$

To evaluate $d_{0,0 ; 0,0}^{\prime 2}$

$$
\begin{aligned}
d_{0,0 ; 0,0}^{\prime} & =<f, \psi_{0,0 ; 0,0}^{\prime}> \\
& =\int_{0}^{3 / 4} \int_{0}^{3 / 4} \psi_{0,0}\left(t_{1}\right) \varphi_{0,0}\left(t_{2}\right) d t_{1} d t_{2} \\
& =\int_{0}^{3 / 4} \psi_{0,0}\left(t_{1}\right) d t_{1} \int_{0}^{3 / 4} \varphi_{0,0}\left(t_{2}\right) d t_{2} \\
& =\frac{3}{2^{4}}
\end{aligned}
$$

Then, $d_{0,0 ; 0,0}^{\prime 2}=\frac{3^{2}}{2^{8}}$

To evaluate $d_{1,1 ; 0,0}^{\prime 2}$

$$
\begin{aligned}
d_{1,1 ; 0,0}^{\prime} & =<f, \psi_{1,1 ; 0,0}^{\prime}> \\
& =\int_{1 / 2}^{3 / 4} \int_{0}^{3 / 4} \psi_{1,1}\left(t_{1}\right) \varphi_{0,0}\left(t_{2}\right) d t_{1} d t_{2} \\
& =\int_{1 / 2}^{3 / 4} \psi_{1,1}\left(t_{1}\right) d t_{1} \int_{0}^{3 / 4} \varphi_{0,0}\left(t_{2}\right) d t_{2} \\
& =\frac{3 * 2^{1 / 2}}{2^{4}}
\end{aligned}
$$

Then, $d_{1,1 ; 0,0}^{\prime 2}=\frac{2 * 3^{2}}{2^{8}}$

To evaluate ${c^{\prime}}_{0,0 ; 0,0}^{2}$

$$
\begin{aligned}
& \because d_{0,0 ; 0,0}^{\prime 2}=\frac{3^{2}}{2^{8}} \\
& \therefore c_{0,0 ; 0,0}^{\prime 2}=\frac{3^{2}}{2^{8}}
\end{aligned}
$$

To evaluate ${c^{\prime}}_{0,0 ; 1,1}^{2}$

$$
\begin{aligned}
& \because d_{1,1 ; 0,0}^{\prime 2}=\frac{2 * 3^{2}}{2^{8}} \\
& \therefore c_{0,0 ; 1,1}^{\prime 2}=\frac{2 * 3^{2}}{2^{8}}
\end{aligned}
$$

To evaluate $d_{0,0 ; 0,0}^{2}$

$$
\begin{aligned}
d_{0,0 ; 0,0} & =<f, \psi_{0,0 ; 0,0}> \\
& =\int_{0}^{3 / 4} \int_{0}^{3 / 4} \psi_{0,0}\left(t_{1}\right) \psi_{0,0}\left(t_{2}\right) d t_{1} d t_{2} \\
& =\int_{0}^{3 / 4} \psi_{0,0}\left(t_{1}\right) d t_{1} \int_{0}^{3 / 4} \psi_{0,0}\left(t_{2}\right) d t_{2} \\
& =\frac{1}{2^{4}} \\
\text { Then, } d_{0,0 ; 0,0}^{2} & =\frac{1}{2^{8}}
\end{aligned}
$$

To evaluate $d_{1,1 ; 0,0}^{2}$

$$
\begin{aligned}
d_{1,1 ; 0,0} & =<f, \psi_{1,1 ; 0,0}> \\
& =\int_{1 / 2}^{3 / 4} \int_{0}^{3 / 4} \psi_{1,1}\left(t_{1}\right) \psi_{0,0}\left(t_{2}\right) d t_{1} d t_{2} \\
& =\int_{1 / 2}^{3 / 4} \psi_{1,1}\left(t_{1}\right) d t_{1} \int_{0}^{3 / 4} \psi_{0,0} d t_{2} \\
& =\frac{2^{1 / 2}}{2^{4}} \\
\text { Then, } d_{1,1 ; 0,0}^{2} & =\frac{2}{2^{8}}
\end{aligned}
$$

To evaluate $d_{0,0 ; 1,1}^{2}$

$$
\begin{aligned}
& \because d_{1,1 ; 0,0}^{2}=\frac{2}{2^{8}} \\
& \therefore d_{0,0 ; 1,1}^{2}=\frac{2}{2^{8}}
\end{aligned}
$$

To evaluate $d_{1,1 ; 1,1}^{2}$

$$
\begin{aligned}
d_{1,1 ; 1,1} & =\left\langle f, \psi_{1,1 ; 1,1}>\right. \\
& =\int_{1 / 2}^{3 / 4} \int_{1 / 2}^{3 / 4} \psi_{1,1}\left(t_{1}\right) \psi_{1,1}\left(t_{2}\right) d t_{1} d t_{2} \\
& =\int_{1 / 2}^{3 / 4} \psi_{1,1}\left(t_{1}\right) d t_{1} \int_{1 / 2}^{3 / 4} \psi_{1,1}\left(t_{2}\right) d t_{2} \\
& =\frac{2}{2^{4}} \\
\text { Then, } d_{1,1 ; 1,1}^{2} & =\frac{4}{2^{8}}
\end{aligned}
$$

rest of the terms will be 0 .

putting these calculated values in equation (35), we get

$$
\begin{aligned}
\text { R.H.S }= & \frac{9^{2}}{2^{8}}+\frac{3^{2}}{2^{8}}+\frac{2 * 3^{2}}{2^{8}}+0+0+\frac{3^{2}}{2^{8}}+\frac{1}{2^{8}}+\frac{2}{2^{8}} \\
& +0+0+\frac{2 * 3^{2}}{2^{8}}+\frac{2}{2^{8}}+\frac{4}{2^{8}}+0+0+0+0 \\
& +0+0+0+0+0+0+0+0 \\
= & \frac{9}{2^{4}}
\end{aligned}
$$


By equation (34) and (36), we get

$$
\text { L.H.S. = R.H.S. }
$$

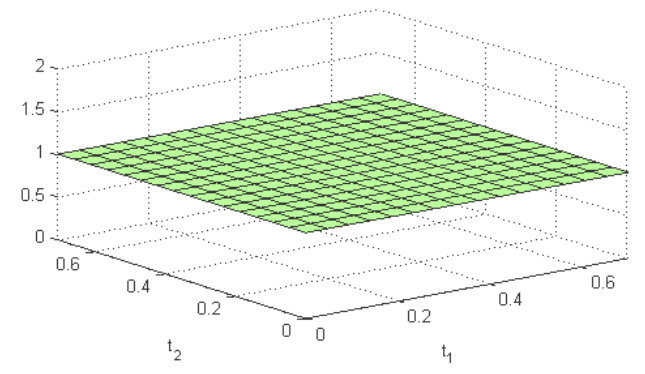

Fig. 21. Graph of signal $f\left(t_{1}, t_{2}\right)=\chi_{[0,3 / 4]}\left(t_{1}\right) \chi_{[0,3 / 4]}\left(t_{2}\right)$ with order of matrix $=16$

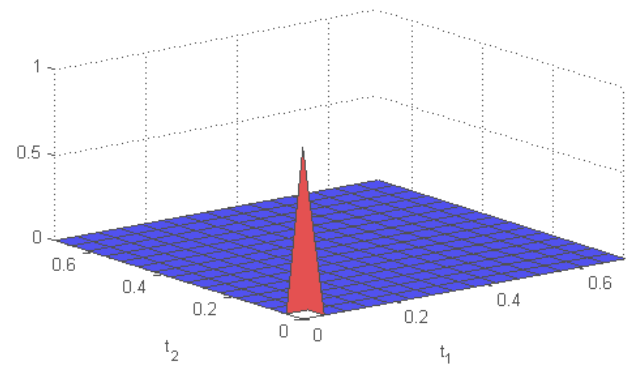

Fig. 22. Graph of decomposed signal $f\left(t_{1}, t_{2}\right)$

$\chi_{[0,3 / 4]}\left(t_{1}\right) \quad \chi_{[0,3 / 4]}\left(t_{2}\right)$ with order of matrix=16 at resolutions $m_{1}=m_{2}=0$

\section{CONCLUSIONS}

(i) In best of our knowledge, the signals considered in this paper have not been studied till now by any researchers of working in signal and image processing. Thus the detailed studies of images of corresponding signals are new and interesting approches in the analysis of signal and image processing.

(ii) The correct double wavelet series of a signal $f$ of two variables $t_{1}$ and $t_{2}$ in $[0,1] \times[0,1]$ by using double Haar Scaling function $\phi\left(t_{1}, t_{2}\right)$ and Haar Wavelet function $\psi\left(t_{1}, t_{2}\right)=\psi\left(t_{1}\right) \psi\left(t_{2}\right)$ is introduced and this expression of signals is verified by a number of examples. This is a significant idea/development of this research paper in signal and image processing by wavelet methods.

\section{ACKNOWLEDGMENTS}

Shyam Lal, one of the authors, is thankful to DST - CIMS for encouragement to this work. Authors are grateful to the referee for his valuable suggestions and comments which improve the presentation of the research paper.

\section{REFERENCES}

[1] Haar A., Zurtheorie der orthogonalen funktionen systeme, Math. Ann., Vol. 69, pp. 331-371, 1910.

[2] Tichmarsh.,E.C., The Theory of Functions, Second Edition, Oxford University Press, (1939). 